\title{
Predictors of mortality, ICU admission and intubation among hospitalized COVD-19 patients in Jordan.
}

\author{
Predictors of COVID-19 Severity and Mortality in Jordan
}

Khaled Al Oweidat ${ }^{1}$, Asma S. Albtoosh ${ }^{1}$, Ahmad A. Toubasi ${ }^{2 *}$, Mona Khaled Ribie ${ }^{3}$, Manar M. Hasuneh ${ }^{2}$, Daniah L.

\section{Alfaqheri ${ }^{2}$, Abdullah H. Alshurafa ${ }^{2}$, Mohammad Ribie ${ }^{2}$, Nathir Obeidat ${ }^{1}$}

${ }^{1}$ Department of Respiratory and Sleep Medicine, Department of Internal Medicine, School of Medicine, The University of Jordan, Amman, Jordan.

${ }^{2}$ Faculty of Medicine, The University of Jordan, Amman, Jordan.

${ }^{3}$ Department of Internal Medicine, School of Medicine, The University of Jordan, Amman, Jordan.

*The corresponding author:

Ahmad A. Toubasi

Faculty of Medicine, the University of Jordan, Amman, 11942, Jordan. 
Email Address: tubasi_ahmad@yahoo.com

Phone Number: 00962798035061

\section{Abstract}

\section{Introduction}

Several studies characterized COVID-19 patients and revealed the factors associated with COVID-19 mortality and severity.

However, few studies were conducted in the Middle East, hence we decided to conduct this study to characterize COVID-19 patients, identify the factors associated with COVID-19 mortality and severity and to compare the patients' outcomes in the two waves of the pandemic.

\section{Method}

We conducted a retrospective single center cohort study among COVID-19 patients admitted to our hospital. The primary outcomes were COVID-19 related death, ICU admission and invasive intubation. Whereas, the secondary outcome was the length of hospital 
stay. In addition, the patients were divided into two cohorts to represent the two waves of the pandemic, to compare between them based on the outcomes.

\section{Results}

The total number of COVID-19 patients included in our study was 745. The overall rates of COVID-19 related mortality, ICU admission and invasive intubation were $23.0 \%, 28.3 \%$ and $10.8 \%$, respectively. $\mathrm{CKD}$, troponin, $\mathrm{LDH}$ and $\mathrm{O} 2$ saturation $<90 \%$ were significant predictors for mortality. Moreover, the significant predictors for ICU admission were HF and remdesivir use. Whereas, O2 saturation $<90 \%$ and GI symptoms were the only significant predictors for invasive intubation. Additionally, the second wave had significantly higher rates of ICU admission compared to the first one.

\section{Conclusion}

Our study showed that several factors predicted COVID-19 mortality, ICU admission and invasive intubation. Additionally, our study revealed that the second wave of the pandemic had significantly higher rates of ICU admission but not mortality or invasive intubation compared to the first wave. 

author/funder, who has granted medRxiv a license to display the preprint in perpetuity. It is made available under a CC-BY 4.0 International license. 


\section{Introduction}

A novel coronavirus $(\mathrm{CoV})$ named severe acute respiratory syndrome coronavirus 2 (SARS-CoV-2) by the World Health Organization (WHO) is responsible for the recent pneumonia pandemic that started in early December, 2019 in Wuhan City, Hubei Province, China $[1,2]$. The transmission of SARS-CoV-2 occurs mainly through respiratory droplets and aerosols and causes a disease that is now referred to as COVID-19 [3]. The disease was classified based on the diverse clinical features it can present with into asymptomatic, mild, moderate, severe and critical. Asymptomatic infection was defined as having no symptoms that are consistent with COVID-19. When individuals presented with the signs and symptoms of COVID-19 without shortness of breath or abnormal chest imaging, the illness was classified as mild. Moderate illness was defined as individuals who show evidence of lower respiratory disease during clinical assessment or imaging and who have an oxygen saturation $\left(\mathrm{SpO}_{2}\right) \geq 94 \%$ on room air at sea level. Severe illness was defined as individuals who have $\mathrm{SpO}_{2}<94 \%$ on room air at sea level, a ratio of arterial partial pressure of oxygen to fraction of inspired oxygen $\left(\mathrm{PaO}_{2} / \mathrm{FiO}_{2}\right)<300 \mathrm{~mm} \mathrm{Hg}$, a respiratory rate $>30$ breaths/min, or lung infiltrates $>50 \%$. Lastly, the definition of critical illness was made in individuals who have respiratory failure, septic shock, and/or multiple organ dysfunction [4].

As of January 2022, over 350 million COVID-19 cases have been reported worldwide resulting in upwards of 5 million deaths [5]. Mortality rate early in the pandemic was high among hospitalized patients approaching 32\% [6]. Published research on COVID-19 disease described many factors that predict severe and fatal COVID-19 disease, including: demographic factors as well as clinical, 
immunological, hematological, biochemical and radiographic findings [7]. Demographic factors such as age and gender have been reported to impact COVID-19 outcomes with older age predicting disease severity and survival [7-11] and male sex associated with a severe clinical course and mortality [8]. Preexisting comorbid conditions such as cardiovascular disease, diabetes, chronic respiratory disease, hypertension, and cancer were also associated with increased case fatality rate [9-15]. Moreover, high levels of C-reactive proteins (CRP), D-dimer, procalcitonin (PCT), total bilirubin, renal dysfunction indices and interleukin-6 level (IL-6) as well as low lymphocyte count were found to predict severe disease course and death [12]. On the other hand, patients who were treated with methylprednisolone had lower mortality rates than patients who were not treated with the drug [12].

Until the $28^{\text {th }}$ of January 2022, Jordan had 13,157 COVID-19 deaths and a total of 1,189,080 cases [5]. In the Middle East, a few studies revealed the clinical characteristics [16] and predictors of COVID-19 severity and mortality [17]. To our best knowledge, no studies were conducted in Jordan to reveal the predictors of COVID-19 mortality and severity. Accordingly, the scarcity of data about COVID-19 in the Middle East necessitates the conduct of studies that characterize COVID-19 patients in the region. The aim of this study was to describe the clinical characteristics of COVID-19 in hospitalized patients and identify risk factors associated with mortality, ICU admission, intubation rate and length of stay in those patients. In addition, our study aimed to compare the outcomes between the two admission waves of the pandemic in our hospital.

\section{Material and Methods}




\section{Study population}

A trained team of physicians and medical students performed a retrospective study among hospitalized COVID-19 patients at Jordan University Hospital (JUH) in Amman, Jordan using the hospital's electronic medical records (EMR). Data was extracted from a total of 753 medical records of COVID-19 patients with a laboratory confirmed diagnosis of SARS-CoV-2 infection by nasopharyngeal swab real-time reverse-transcriptase polymerase chain reaction (RT-PCR). The RT-PCR was performed using Zybio nucleic acid extraction test, China and the detection kit was Zybio nucleic acid detection kit. The RT-PCR samples were collected within the first two weeks of the symptoms' onset. All patients who were admitted to JUH between September 2020 to August 2021 were eligible for inclusion in the study. The patients were divided into two cohorts to represent the two waves of the pandemic in Jordan and, to subsequently compare between them based on the outcome measures. The first wave of the pandemic in Jordan was from September, 2020 to January, 2021 while the second wave was between February, 2021 and August, 2021.

\section{Data Collection}

The following patients' data was collected:

- Patient's characteristics, including: demographics (age, sex and smoking status), presenting symptoms (cough, shortness of breath, runny nose, fever, headache and gastrointestinal symptoms) and comorbidities (hypertension (HTN), diabetes mellitus 
(DM), cardiovascular disease (CVD), heart failure (HF), chronic kidney disease (CKD), malignancy, asthma, chronic obstructive pulmonary disease (COPD), dyslipidemia, neurological diseases and autoimmune diseases). In addition, patients' presentation with fever or hypoxia was documented. Presence of fever on admission was identified by an emergency room triage oral temperature higher than $37.7^{\circ} \mathrm{C}$ [18], with hypoxia on admission determined by oxygen saturation and subsequently divided into 3 groups; $<90 \%, 90 \%-94 \%$, and $>94 \%$ on room air or ambient air.

- Patient's venous blood sample laboratory results were collected. These included complete blood count (CBC), D-dimer, Creactive protein (CRP), Interleukin 6 (IL6), Troponin I, Procalcitonin, absolute lymphocyte count, ferritin level, serum creatinine, urea, last hemoglobin A1c (HbA1C) within 3 months of admission. In addition, lactate dehydrogenase (LDH), aspartate aminotransferase (AST), alanine aminotransferase (ALT), albumin, serum potassium, and serum sodium levels were also collected. Serum creatinine values were used to identify Acute kidney injury (AKI) among COVID-19 patients which was defined as an increase in serum creatinine to $\geq 1.5$ times the baseline [19]. Moreover, troponin values were considered positive if they were $\geq 45 \mathrm{pg} / \mathrm{ml}$. Furthermore, the $\mathrm{HbA} 1 \mathrm{C}$ within the last 3 months of the admission was used to determine the state of diabetes control among patients with diabetes, with an $\mathrm{HbA} 1 \mathrm{C} \geq 9$ indicating uncontrolled diabetes.

- Radiological features, including chest x-ray, high-resolution computed tomography (HRCT) and computed tomography pulmonary angiogram (CTPA). 
- In-hospital management details, including medication received during admission (dexamethasone, antiviral therapy, tocilizumab) and oxygen delivery devices such as nasal cannula, face mask, venturi, non-rebreather mask, non-invasive ventilation, high flow nasal cannula and invasive mechanical ventilation.

- Patient's outcomes during the entire hospitalization period including death, ICU admission, intubation and length of stay.

\section{Outcome Measures}

The primary outcomes were COVID-19 related death, ICU admission and invasive intubation. Whereas, the secondary outcome was the length of hospital stay. COVID-19 related death was defined as mortality that occurred within the first 28 days from the admission and was attributed to one of the COVID-19 complications.

\section{Ethical Statement}

Given the retrospective nature of the study, informed consent was not required. The study protocol was approved by the Institutional Review Board at JUH.

\section{Statistical Analysis}

The patients' data was entered on Microsoft Office Excel 2019, then imported into IBM SPSS v.25 software which was used to conduct the analysis. Counts and percentages were used to describe categorical variables whereas means and standard deviations were 
used to describe continuous variables. To identify the predictors of COVID-19 related mortality, ICU admission and invasive intubation, univariate binary logistic regression analysis was used. In addition, univariate linear regression was used to identify the predictors of length of hospital stay. To adjust for confounding variables, significant predictors of COVID-19 related mortality, ICU admission and invasive intubation were reexamined using multivariate binary logistic regression. While significant predictors of length of hospital stay were retested using multivariate linear regression. Results of univariate binary and linear logistic regression were expressed using crude odds ratio (COR) and crude B coefficient (CB) with their corresponding 95\% confidence intervals (95\% CIs), respectively. While the results of the multivariate binary and linear logistic regression models were expressed using adjusted odds ratio (AOR) and adjusted B coefficient (AB) with their corresponding 95\% confidence intervals (95\% CIs), respectively. All the variables with a P-value $<0.05$ in the univariate and multivariate logistic regression models were considered statistically significant.

\section{Results}

\section{General Characteristics of the Included Patients}

We retrieved 753 medical records of COVID-19 positive patients admitted in our hospital, of which 8 records were excluded due to missing data about their outcomes. The total number of the COVID-19 patients included in our study was 745 patients. The detailed characteristics of the patients are described in Table 1 . The percentage of males in the study was $51.3 \%(382 / 745)$. The mean of the patients' age was $63.15 \pm 15.99$ and the majority of the patients were non-smokers $(86.6 \%)$. Moreover, the most frequent chief 
complaints reported by the patients were shortness of breath (35.0\%), cough (15.5\%) and generalized weakness (15.0\%). In addition, $30.4 \%$ of the patients reported gastrointestinal symptoms (226/744). Regarding comorbidities, $44.8 \%$ of the patients had Diabetes Mellitus (DM) and $55.1 \%$ had Hypertension (HTN). Furthermore, $16.1 \%$ of the patients had coronary artery disease (CAD) and $8 \%$ of the patients had heart failure (HF). Additionally, $12.5 \%$ and $5.9 \%$ of the patients had chronic kidney disease (CKD) and neurologic diseases, respectively. Moreover, only $3.2 \%$ of the patients were vaccinated and the majority (64.4\%) of the patients were from the second wave of the pandemic.

Table 1. The General Demographics of the Patients

\begin{tabular}{|c|c|c|c|}
\hline Variable & Response & $\begin{array}{c}\text { Frequency } \\
(n=745)\end{array}$ & Percentage (\%) \\
\hline \multirow{2}{*}{ Sex } & Male & 382 & 51.3 \\
\hline & Female & 363 & 48.7 \\
\hline \multirow{3}{*}{ Smoking Status } & Non-Smoker & 645 & 86.6 \\
\hline & Smoker & 60 & 8.1 \\
\hline & Ex-Smoker & 40 & 5.4 \\
\hline Chief Complaint & Generalized Weakness & 110 & 15.0 \\
\hline
\end{tabular}




\begin{tabular}{|c|c|c|c|}
\hline Variable & Response & $\begin{array}{l}\text { Frequency } \\
(n=745)\end{array}$ & Percentage (\%) \\
\hline & Shortness of Breath & 256 & 35.0 \\
\hline & Cough & 113 & 15.5 \\
\hline & Fever & 89 & 12.2 \\
\hline & Others & 71 & 9.7 \\
\hline & GI symptoms & 50 & 6.8 \\
\hline & Chest pain & 27 & 3.7 \\
\hline & Asymptomatic & 4 & 0.5 \\
\hline \multirow{2}{*}{ Fatigue } & No & 372 & 50.4 \\
\hline & Yes & 366 & 49.6 \\
\hline \multirow{2}{*}{ Fever } & No & 390 & 52.8 \\
\hline & Yes & 348 & 47.2 \\
\hline \multirow{2}{*}{ Chills } & No & 473 & 64.1 \\
\hline & Yes & 265 & 35.9 \\
\hline
\end{tabular}




\begin{tabular}{|c|c|c|c|}
\hline Variable & Response & $\begin{array}{c}\text { Frequency } \\
(n=745)\end{array}$ & Percentage $(\%)$ \\
\hline \multirow{2}{*}{ GI Symptoms } & No & 518 & 69.6 \\
\hline & Yes & 226 & 30.4 \\
\hline \multirow{2}{*}{ Headache } & No & 662 & 89.0 \\
\hline & Yes & 82 & 11.0 \\
\hline \multirow{2}{*}{ Nasal Discharge } & No & 710 & 95.4 \\
\hline & Yes & 34 & 4.6 \\
\hline \multirow{2}{*}{ Sore Throat } & No & 684 & 91.9 \\
\hline & Yes & 60 & 8.1 \\
\hline \multirow{2}{*}{ Chest Pain } & No & 537 & 72.2 \\
\hline & Yes & 207 & 27.8 \\
\hline \multirow{2}{*}{ Shortness of Breath } & No & 289 & 38.8 \\
\hline & Yes & 455 & 61.2 \\
\hline Cough & No & 247 & 33.4 \\
\hline
\end{tabular}




\begin{tabular}{|c|c|c|c|}
\hline Variable & Response & $\begin{array}{l}\text { Frequency } \\
(n=745)\end{array}$ & Percentage (\%) \\
\hline & Yes & 492 & 66.6 \\
\hline \multirow{2}{*}{ Diabetes Mellitus } & No & 412 & 55.2 \\
\hline & Yes & 334 & 44.8 \\
\hline \multirow{2}{*}{ Hypertension } & No & 335 & 44.9 \\
\hline & Yes & 411 & 55.1 \\
\hline \multirow{2}{*}{ Coronary Artery Disease } & No & 626 & 83.9 \\
\hline & Yes & 120 & 16.1 \\
\hline \multirow{2}{*}{ Heart Failure } & No & 686 & 92.0 \\
\hline & Yes & 60 & 8.0 \\
\hline \multirow{2}{*}{ Other Cardiovascular Diseases } & No & 716 & 96.0 \\
\hline & Yes & 30 & 4.0 \\
\hline \multirow{2}{*}{ Asthma } & No & 706 & 94.6 \\
\hline & Yes & 40 & 5.4 \\
\hline
\end{tabular}




\begin{tabular}{|c|c|c|c|}
\hline Variable & Response & $\begin{array}{l}\text { Frequency } \\
(n=745)\end{array}$ & Percentage (\%) \\
\hline \multirow{2}{*}{$\begin{array}{c}\text { Chronic Obstructive Pulmonary } \\
\text { Diseases }\end{array}$} & No & 723 & 96.9 \\
\hline & Yes & 23 & 3.1 \\
\hline \multirow{2}{*}{ Other Respiratory Diseases } & No & 674 & 90.3 \\
\hline & Yes & 72 & 9.7 \\
\hline \multirow{2}{*}{ Autoimmune Diseases } & No & 703 & 94.2 \\
\hline & Yes & 43 & 5.8 \\
\hline \multirow{2}{*}{ Chronic Kidney Diseases } & No & 653 & 87.5 \\
\hline & Yes & 93 & 12.5 \\
\hline \multirow{2}{*}{ Dyslipidemia } & No & 729 & 97.7 \\
\hline & Yes & 17 & 2.3 \\
\hline \multirow{2}{*}{ Cancer } & No & 713 & 95.6 \\
\hline & Yes & 33 & 4.4 \\
\hline Neurologic Diseases & No & 702 & 94.1 \\
\hline
\end{tabular}




\begin{tabular}{|c|c|c|c|}
\hline Variable & Response & $\begin{array}{c}\text { Frequency } \\
(n=745)\end{array}$ & Percentage $(\%)$ \\
\hline & Yes & 44 & 5.9 \\
\hline \multirow{2}{*}{ Pregnancy } & No & 356 & 98.1 \\
\hline & Yes & 7 & 1.9 \\
\hline \multirow{2}{*}{ Vaccination Status } & Not vaccinated & 721 & 96.8 \\
\hline & Vaccinated & 24 & 3.2 \\
\hline \multirow{2}{*}{ Wave } & First & 265 & 35.6 \\
\hline & Second & 480 & 64.4 \\
\hline \multirow{2}{*}{ Admission } & Floor & 524 & 71.7 \\
\hline & ICU & 207 & 28.3 \\
\hline \multirow{3}{*}{ Outcome } & Discharge & 547 & 74.7 \\
\hline & COVID-19 related death & 171 & 23.0 \\
\hline & Non COVID-19 related death & 14 & 2.3 \\
\hline Outcome among ICU patients & Discharge & 97 & 46.9 \\
\hline
\end{tabular}




\begin{tabular}{|c|c|c|c|c|}
\hline Variable & \multicolumn{2}{|c|}{ Response } & $\begin{array}{c}\text { Frequency } \\
(n=745)\end{array}$ & Percentage (\%) \\
\hline & \multicolumn{2}{|c|}{ COVID-19 related death } & 110 & 53.1 \\
\hline & Variable & Mean & \multicolumn{2}{|c|}{ Standard Deviation } \\
\hline & Age & 63.149 & & 15.99 \\
\hline \multicolumn{2}{|r|}{ Length of Hospital Stay } & 10.09 & & 9.08 \\
\hline
\end{tabular}

\section{Investigations and Treatment Methods}

The $\mathrm{O} 2$ saturation on admission was $90 \%-94 \%$ in $24.4 \%$ of the patients and $<90 \%$ in $41.9 \%$ of them while only $22.5 \%$ of the patients had documented fever. The most frequently used imaging methods were chest x-ray, High Resolution Computed Tomography (HRCT) and Computed Tomography Pulmonary Angiography (CTPA). The most frequently observed changes on chest x-ray was bilateral infiltrates $(70.9 \%)$ whereas, the most frequently observed changes on HRCT was ground glass opacifications (70.0\%).

Additionally, $14.0 \%$ had a positive CTPA indicating Pulmonary Embolism (PE). Regarding the laboratory investigations, troponin and D-dimer were positive in $21.1 \%$ and $85.7 \%$ of the patients, respectively. Additionally, creatinine was higher than the cut-off point for the definition of Acute Kidney Injury (AKI) in 30.5\% of the patients. Whereas, the mean and standard deviation of hemoglobin 
$(\mathrm{Hb})$ and white blood cells (WBC) count were 12.45 \pm 2.21 and 10.84E3 $\pm 39.31 \mathrm{E} 3$, respectively. Moreover, the mean and standard deviation of the ferritin and lactate dehydrogenase (LDH) were 593.25 \pm 583.143 and $701.91 \pm 633.93$, respectively. The most frequently used O2 delivery device was nasal cannula as it was used in $49.7 \%$ of the patients. The second and the third most frequently used $\mathrm{O} 2$ delivery devices were the simple face mask (27.3\%) and non-rebreather mask (24.5\%), respectively. Moreover, regarding medications used in treating COVID-19 patients, systemic corticosteroids were used in $88.6 \%$ of the patients, remdesivir in $24.6 \%$ and tocilizumab in $9.1 \%$ of them. The detailed data about the investigations and treatment methods of the patients are described in Table 2.

Table 2. The Investigations and Treatment methods of the Patients

\begin{tabular}{|c|c|c|c|}
\hline Variable & Response & Frequency $(n=745)$ & Percentage $(\%)$ \\
\hline \multirow{3}{*}{ Chest X-ray } & Clear & 82 & 11.7 \\
\hline & Unilateral Infiltrate & 122 & 17.4 \\
\hline & Bilateral Infiltrate & 496 & 70.9 \\
\hline \multirow{3}{*}{ High Resolution Computed Tomography } & Clear & 5 & 5.0 \\
\hline & Ground Glass & 70 & 70.0 \\
\hline & Fibrotic Changes & 25 & 25.0 \\
\hline \multirow{2}{*}{ Computed Tomography Pulmonary Angiography } & Negative & 37 & 86.0 \\
\hline & Positive & 6 & 14.0 \\
\hline
\end{tabular}




\begin{tabular}{|c|c|c|c|}
\hline \multirow{2}{*}{ Troponin } & Negative & 377 & 78.9 \\
\hline & Positive & 101 & 21.1 \\
\hline \multirow{2}{*}{ D-Dimer } & Negative & 91 & 14.3 \\
\hline & Positive & 546 & 85.7 \\
\hline \multirow{2}{*}{ Acute Kidney Injury (Creatinine) } & Negative & 511 & 69.5 \\
\hline & Positive & 224 & 30.5 \\
\hline \multirow{2}{*}{ Last HBA1C } & Controlled & 190 & 87.6 \\
\hline & Uncontrolled & 27 & 12.4 \\
\hline \multirow{3}{*}{ O2 Saturation } & $>=94$ & 239 & 33.7 \\
\hline & $90-94$ & 173 & 24.4 \\
\hline & $<90$ & 297 & 41.9 \\
\hline \multirow{2}{*}{ Documented Fever } & No & 578 & 77.5 \\
\hline & Yes & 168 & 22.5 \\
\hline \multirow{6}{*}{ Oxygen Delivery Devices } & None & 133 & 18.0 \\
\hline & Nasal Canula & 368 & 49.7 \\
\hline & Face Mask & 202 & 27.3 \\
\hline & Venturi & 36 & 4.9 \\
\hline & Non-Rebreather Mask & 181 & 24.5 \\
\hline & $\begin{array}{l}\text { Non-invasive } \\
\text { Ventilation }\end{array}$ & 118 & 15.9 \\
\hline
\end{tabular}




\begin{tabular}{|c|c|c|c|}
\hline & High Flow Nasal Canula & 129 & 17.4 \\
\hline & Intubation & 80 & 10.8 \\
\hline \multirow{3}{*}{ Treatments Used } & Steroids & 656 & 88.6 \\
\hline & Tocilizumab & 68 & 9.1 \\
\hline & Remdesivir & 183 & 24.6 \\
\hline Variable & \multicolumn{2}{|c|}{ Mean } & Standard Deviation \\
\hline IL-6 (pg/ml) & \multicolumn{2}{|c|}{41.21} & 63.85 \\
\hline Brain Natriuretic Peptide (pg/ml) & \multicolumn{2}{|c|}{445.62} & 1122.70 \\
\hline Pro-calcitonin (ng/ml) & \multicolumn{2}{|c|}{1.37} & 5.47 \\
\hline Hemoglobin $(\mathrm{g} / \mathrm{dl})$ & \multicolumn{2}{|c|}{12.45} & 2.21 \\
\hline White Blood Cells Count & \multicolumn{2}{|c|}{$10.84 \mathrm{E} 3$} & 39.31E3 \\
\hline Neutrophils Absolute Count & \multicolumn{2}{|c|}{$8.63 \mathrm{E} 3$} & $34.75 \mathrm{E} 3$ \\
\hline Lymphocyte Absolute Count & \multicolumn{2}{|c|}{$1.33 \mathrm{E} 3$} & $3.46 \mathrm{E} 3$ \\
\hline Platelets Count & \multicolumn{2}{|c|}{$273.45 \mathrm{E} 3$} & $272.11 \mathrm{E} 3$ \\
\hline Variable & \multicolumn{2}{|c|}{ Mean } & Standard Deviation \\
\hline C-Reactive Protein (mg/l) & \multicolumn{2}{|c|}{132.60} & 567.24 \\
\hline Ferritin (ng/ml) & \multicolumn{2}{|c|}{593.25} & 583.14 \\
\hline Lactate Dehydrogenase $(\mathrm{u} / \mathrm{l})$ & \multicolumn{2}{|c|}{701.91} & 633.93 \\
\hline
\end{tabular}




\begin{tabular}{|c|c|c|}
\hline Potassium (mg/dl) & 4.71 & 1.8 \\
\hline Sodium (mg/dl) & 129.40 & 30.68 \\
\hline Urea $(\mathrm{mg} / \mathrm{dl})$ & 54.13 & 36.73 \\
\hline Alanine Transaminase $(\mathrm{u} / \mathrm{l})$ & 37.12 & 114.54 \\
\hline Aspartate Transaminase $(\mathrm{u} / \mathrm{l})$ & 51.23 & 171.99 \\
\hline Total Bilirubin mg/dl & 0.78 & 1.31 \\
\hline Direct Bilirubin mg/dl & 8.37 & 25.433 \\
\hline
\end{tabular}

\section{Predictors of COVID-19 Outcomes}

Regarding the outcomes of COVID-19 patients, $23.0 \%$ of all the patients died (COVID-19 related death) while $53.1 \%$ of the patients who were admitted to ICU died (Table 1). In the non-adjusted model, age, DM, HTN, CAD,HF CKD, neurologic diseases, chest xray patterns, troponin, hemoglobin, WBCs, neutrophils, D-dimer, AKI, LDH, Potassium, total bilirubin and $\mathrm{O} 2$ saturation $<90 \%$ were significant predictors for mortality. After adjustment for confounding variables, CKD (AOR=3.831;95\% CI: 1.179-12.446), troponin $(\mathrm{AOR}=3.060 ; 95 \% \mathrm{CI}: 1.156-8.102), \mathrm{LDH}(\mathrm{AOR}=1.002 ; 95 \% \mathrm{CI}: 1.001-1.003)$ and $\mathrm{O} 2$ saturation $<90 \%(\mathrm{AOR}=2.761 ; 95 \% \mathrm{CI}: 1.066-$ 7.155) were the only significant predictors for mortality (Table 3). Patients who had CKD, positive troponin, high LDH values and O2 saturation $<90 \%$ had a significantly higher risk of mortality. Additionally, regarding COVID-19 severity, $28.3 \%$ of the patients were 
admitted to the ICU and $10.8 \%$ of the patients were intubated (Table 1). In the non-adjusted model that investigated ICU admission, age, smoking status, GI symptoms, DM, HTN, CKD, HF, other CVDs, chest x-ray patterns, troponin, GI symptoms, neutrophils, Ddimer, AKI, potassium, sodium, $\mathrm{O} 2$ saturation $<90 \%$, Tocilizumab use and remdesivir use were significant predictors for ICU admission. However, in the adjusted model, only HF (AOR=7.894;95\% CI: 1.391-44.818) and remdesivir use (AOR=0.192;95\% CI: 0.044-0.835) were significant predictors for ICU admission (Table 3). Patients who had HF had significantly higher risk for ICU admission while patients who were treated using remdesivir had significantly lower risk for ICU admission. Furthermore, in the nonadjusted model that evaluated invasive intubation, GI symptoms, CKD, neurological diseases, chest x-ray patterns, troponin, D-dimer, AKI, sodium, ALT, AST, O2 saturation $<90 \%$ and steroids were significant predictors for invasive intubation. In the adjusted model, O2 saturation $<90 \%(\mathrm{AOR}=16.585 ; 95 \% \mathrm{CI}: 2.892-95.118)$ and GI symptoms (AOR=0.323;95\% CI: $0.105-0.993)$ were the only significant predictors for invasive intubation (Table 3). Patients who had $\mathrm{O} 2$ saturation $<90 \%$ had significantly higher risk for invasive intubation whereas patients who reported GI symptoms had significantly lower risk for it. In regards to the length of hospital stay, the mean and standard deviation for hospital stay was $10.09 \pm 9.08$ (Table 1). In the non-adjusted model, pregnancy, chest x-ray patterns, $\mathrm{Hb}$, D-dimer, total bilirubin, $\mathrm{O} 2$ saturation, Tocilizumab use and remdesivir use were significant predictors for the length of hospital stay. However, after adjusting for confounding variables, none of the aforementioned variables were significant predictors for the length of hospital stay (Table 3). 
Table 3. Regression Analysis for the Predictors of COVID-19 Outcomes

\begin{tabular}{|c|c|c|c|c|c|c|c|c|c|}
\hline \multirow[b]{2}{*}{ Variable } & \multirow[b]{2}{*}{ Response } & \multicolumn{2}{|c|}{ COVID-19 Related Mortality } & \multicolumn{2}{|c|}{ ICU Admission } & \multicolumn{2}{|c|}{ Intubation } & \multicolumn{2}{|c|}{ Length of Hospital Stay } \\
\hline & & $\begin{array}{c}\text { COR }(95 \% \\
\text { CI })\end{array}$ & $\operatorname{AOR}(95 \% \mathrm{CI})$ & $\begin{array}{c}\text { COR }(95 \% \\
\text { CI })\end{array}$ & $\begin{array}{c}\text { AOR }(95 \% \\
\text { CI) }\end{array}$ & $\operatorname{COR}(95 \% \mathrm{CI})$ & $\operatorname{AOR}(95 \%$ CI) & CB $(95 \%$ CI $)$ & AB $(95 \%$ CI $)$ \\
\hline Age & - & $\begin{array}{c}1.047 \\
(1.033- \\
1.061)^{*}\end{array}$ & $\begin{array}{c}1.013 \\
(0.984-1.042)\end{array}$ & $\begin{array}{c}1.034 \\
(1.021-1.046) \\
*\end{array}$ & $\begin{array}{c}0.983 \\
(0.936- \\
1.033)\end{array}$ & $\begin{array}{c}1.012 \\
(0.997-1.028)\end{array}$ & - & $\begin{array}{c}0.015 \\
(-0.027-0.057)\end{array}$ & - \\
\hline \multirow{2}{*}{ Gender } & Female & $\mathrm{R}$ & $\mathrm{R}$ & $\mathrm{R}$ & $\mathrm{R}$ & $\mathrm{R}$ & $\mathrm{R}$ & $\mathrm{R}$ & $\mathrm{R}$ \\
\hline & Male & $\begin{array}{c}1.067 \\
(0.764-1.489)\end{array}$ & - & $\begin{array}{c}0.968 \\
(0.702-1.336)\end{array}$ & - & $\begin{array}{c}1.121 \\
(0.704-1.785)\end{array}$ & - & $\begin{array}{c}-1.158 \\
(-2.478-0.162)\end{array}$ & - \\
\hline \multirow{3}{*}{ Smoking Status } & Non-Smoker & $\mathrm{R}$ & $\mathrm{R}$ & $\mathrm{R}$ & $\mathrm{R}$ & $\mathrm{R}$ & $\mathrm{R}$ & $\mathrm{R}$ & - \\
\hline & Smoker & $\begin{array}{c}0.523 \\
(0.252-1.088)\end{array}$ & - & $\begin{array}{c}0.643 \\
(0.333-1.238)\end{array}$ & $\begin{array}{c}0.796 \\
(0.088- \\
7.169)\end{array}$ & $\begin{array}{c}1.168 \\
(0.510-2.676)\end{array}$ & - & \multirow{2}{*}{$\begin{array}{c}0.945 \\
(-0.799-1.789)\end{array}$} & - \\
\hline & Ex-Smoker & $\begin{array}{c}1.566 \\
(0.798-3.071)\end{array}$ & - & $\begin{array}{c}2.203 \\
(1.147-4.234) \\
*\end{array}$ & $\begin{array}{c}1.174 \\
(0.077- \\
17.827)\end{array}$ & $\begin{array}{c}2.212 \\
(0.978-5.002)\end{array}$ & - & & - \\
\hline Chief Complaints & Fatigue & $\mathrm{R}$ & $\mathrm{R}$ & $\mathrm{R}$ & $\mathrm{R}$ & $\mathrm{R}$ & $\mathrm{R}$ & $\begin{array}{c}0.064 \\
(-0.296-0.425)\end{array}$ & - \\
\hline
\end{tabular}




\begin{tabular}{|c|c|c|c|c|c|c|c|c|c|}
\hline \multirow[b]{2}{*}{ Variable } & \multirow[b]{2}{*}{ Response } & \multicolumn{2}{|c|}{ COVID-19 Related Mortality } & \multicolumn{2}{|c|}{ ICU Admission } & \multicolumn{2}{|c|}{ Intubation } & \multicolumn{2}{|c|}{ Length of Hospital Stay } \\
\hline & & $\begin{array}{c}\text { COR }(95 \% \\
\text { CI) }\end{array}$ & $\operatorname{AOR}(95 \% \mathrm{CI})$ & $\begin{array}{c}\text { COR }(95 \% \\
\text { CI })\end{array}$ & $\begin{array}{c}\text { AOR }(95 \% \\
\text { CI) }\end{array}$ & $\operatorname{COR}(95 \% \mathrm{CI})$ & $\operatorname{AOR}(95 \% \mathrm{CI})$ & CB $(95 \% \mathrm{CI})$ & $\mathrm{AB}(95 \% \mathrm{CI})$ \\
\hline & SOB & $\begin{array}{c}0.772 \\
(0.471-1.265)\end{array}$ & $\begin{array}{c}0.572 \\
(0.201-1.633)\end{array}$ & $\begin{array}{c}1.115 \\
(0.680-1.828)\end{array}$ & - & $\begin{array}{c}1.143 \\
(0.578-2.261)\end{array}$ & - & & \\
\hline & Cough & $\begin{array}{c}0.544 \\
(0.293-1.010)\end{array}$ & $\begin{array}{c}0.433 \\
(0.112-1.671)\end{array}$ & $\begin{array}{c}0.762 \\
(0.416-1.393)\end{array}$ & - & $\begin{array}{c}0.658 \\
(0.269-1.610)\end{array}$ & - & & \\
\hline & Fever & $\begin{array}{c}0.372 \\
(0.182-0.761)\end{array}$ & $\begin{array}{c}0.824 \\
(0.231-2.937)\end{array}$ & $\begin{array}{c}0.888 \\
(0.473-1.669)\end{array}$ & - & $\begin{array}{c}0.539 \\
(0.196-1.482)\end{array}$ & - & & \\
\hline & Atypical & $\begin{array}{c}1.183 \\
(0.627-2.230)\end{array}$ & $\begin{array}{c}1.013 \\
(0.220-4.670)\end{array}$ & $\begin{array}{c}1.216 \\
(0.625-2.366)\end{array}$ & - & $\begin{array}{c}1.368 \\
(0.576-3.249)\end{array}$ & - & & \\
\hline & GI Symptoms & $\begin{array}{c}0.558 \\
(0.250-1.248)\end{array}$ & $\begin{array}{c}0.484 \\
(0.111-2.120)\end{array}$ & $\begin{array}{c}0.920 \\
(0.431-1.964)\end{array}$ & - & $\begin{array}{c}0.318 \\
(0.069-1.465)\end{array}$ & - & & \\
\hline & Chest Pain & $\begin{array}{c}0.622 \\
(0.230-1.680)\end{array}$ & $\begin{array}{c}0.928 \\
(0.163-5.280)\end{array}$ & $\begin{array}{c}0.765 \\
(0.281-2.083)\end{array}$ & - & $\begin{array}{c}0.597 \\
(0.126-2.819)\end{array}$ & - & & \\
\hline
\end{tabular}




\begin{tabular}{|c|c|c|c|c|c|c|c|c|c|}
\hline \multirow[b]{2}{*}{ Variable } & \multirow[b]{2}{*}{ Response } & \multicolumn{2}{|c|}{ COVID-19 Related Mortality } & \multicolumn{2}{|c|}{ ICU Admission } & \multicolumn{2}{|c|}{ Intubation } & \multicolumn{2}{|c|}{ Length of Hospital Stay } \\
\hline & & $\begin{array}{l}\text { COR }(95 \% \\
\text { CI) }\end{array}$ & AOR $(95 \%$ CI) & $\begin{array}{c}\text { COR }(95 \% \\
\text { CI) }\end{array}$ & $\begin{array}{l}\text { AOR }(95 \% \\
\text { CI) }\end{array}$ & $\operatorname{COR}(95 \% \mathrm{CI})$ & $\operatorname{AOR}(95 \% \mathrm{CI})$ & CB $(95 \%$ CI) & $\mathrm{AB}(95 \% \mathrm{CI})$ \\
\hline & Asymptomatic & - & - & - & - & - & - & & \\
\hline & Headache & $\begin{array}{c}0.544 \\
(0.059-5.053)\end{array}$ & - & $\begin{array}{l}1.699 \\
(0.271- \\
10.663)\end{array}$ & - & $\begin{array}{c}1.865 \\
(0.193-17.992)\end{array}$ & - & & \\
\hline & Runny Nose & - & - & - & - & - & - & & \\
\hline \multirow{2}{*}{ GI Symptoms } & No & $\mathrm{R}$ & $\mathrm{R}$ & $\mathrm{R}$ & $\mathrm{R}$ & $\mathrm{R}$ & $\mathrm{R}$ & $\mathrm{R}$ & $\mathrm{R}$ \\
\hline & Yes & $\begin{array}{c}0.722 \\
(0.495-1.052)\end{array}$ & - & $\begin{array}{c}0.584 \\
(0.402-0.848) \\
*\end{array}$ & $\begin{array}{c}0.316 \\
(0.056- \\
1.791)\end{array}$ & $\begin{array}{c}0.538 \\
(0.304-0.954)\end{array}$ & $\begin{array}{c}0.323 \\
(0.105-0.993)^{*}\end{array}$ & $\begin{array}{c}-1.260 \\
(-2.697-0.178)\end{array}$ & - \\
\hline Diabetes Mellitus & - & $\begin{array}{c}1.497 \\
(1.071-2.092) \\
*\end{array}$ & $\begin{array}{c}1.220 \\
(0.567-2.623)\end{array}$ & $\begin{array}{c}1.967 \\
(1.420-2.724) \\
*\end{array}$ & $\begin{array}{c}1.136 \\
(0.299- \\
4.310)\end{array}$ & $\begin{array}{c}1.177 \\
(0.740-1.873)\end{array}$ & - & $\begin{array}{c}0.766 \\
(-0.562-2.094)\end{array}$ & - \\
\hline Hypertension & - & $\begin{array}{c}1.738 \\
(1.229-2.457) \\
*\end{array}$ & $\begin{array}{c}1.123 \\
(0.484-2.606)\end{array}$ & $\begin{array}{c}1.675 \\
(1.201-2.336) \\
*\end{array}$ & $\begin{array}{c}.638 \\
(0.126- \\
3.227)\end{array}$ & $\begin{array}{c}1.093 \\
(0.684-1.748)\end{array}$ & - & $\begin{array}{c}0.994 \\
(-0.335-2.323)\end{array}$ & - \\
\hline
\end{tabular}




\begin{tabular}{|c|c|c|c|c|c|c|c|c|c|}
\hline & & \multicolumn{2}{|c|}{ COVID-19 Related Mortality } & \multicolumn{2}{|c|}{ ICU Admission } & \multicolumn{2}{|c|}{ Intubation } & \multicolumn{2}{|c|}{ Length of Hospital Stay } \\
\hline Variable & Response & $\begin{array}{c}\text { COR }(95 \% \\
\text { CI) }\end{array}$ & $\operatorname{AOR}(95 \% \mathrm{CI})$ & $\begin{array}{c}\text { COR }(95 \% \\
\text { CI })\end{array}$ & $\begin{array}{c}\text { AOR }(95 \% \\
\text { CI) }\end{array}$ & $\operatorname{COR}(95 \% \mathrm{CI})$ & AOR $(95 \% \mathrm{CI})$ & CB $(95 \% \mathrm{CI})$ & $\mathrm{AB}(95 \% \mathrm{CI})$ \\
\hline $\begin{array}{c}\text { Coronary Artery } \\
\text { Disease }\end{array}$ & - & $\begin{array}{c}1.582 \\
(1.033-2.423) \\
*\end{array}$ & $\begin{array}{c}0.734 \\
(0.308-1.752)\end{array}$ & $\begin{array}{c}1.066 \\
(0.692-1.642)\end{array}$ & - & $\begin{array}{c}0.634 \\
(0.308-1.306)\end{array}$ & - & $\begin{array}{c}0.781 \\
(-1.006-2.568)\end{array}$ & - \\
\hline $\begin{array}{c}\text { Chronic Kidney } \\
\text { Disease }\end{array}$ & - & $\begin{array}{c}3.501 \\
(2.229-5.499) \\
*\end{array}$ & $\begin{array}{c}3.831 \\
(1.179-12.446)^{*}\end{array}$ & $\begin{array}{c}2.542 \\
(1.627-3.972) \\
*\end{array}$ & $\begin{array}{c}0.637 \\
(0.077- \\
5.306)\end{array}$ & $\begin{array}{c}2.074 \\
(1.153-3.728)^{*}\end{array}$ & $\begin{array}{c}3.640 \\
(0.895-14.802)\end{array}$ & $\begin{array}{c}-0.395 \\
(-2.384-1.595)\end{array}$ & - \\
\hline Asthma & - & $\begin{array}{c}0.851 \\
(0.397-1.823)\end{array}$ & - & $\begin{array}{c}0.748 \\
(0.349-1.605)\end{array}$ & - & $\begin{array}{c}0.656 \\
(0.198-2.178)\end{array}$ & - & $\begin{array}{c}0.011 \\
(-2.961-2.984)\end{array}$ & - \\
\hline COPD & - & $\begin{array}{c}0.434 \\
(0.128-1.479)\end{array}$ & - & $\begin{array}{c}0.890 \\
(0.346-2.290)\end{array}$ & - & $\begin{array}{c}1.776 \\
(0.589-5.356)\end{array}$ & - & $\begin{array}{c}-2.163 \\
(-5.940-1.613)\end{array}$ & - \\
\hline Dyslipidemia & - & $\begin{array}{c}0.628 \\
(0.178-2.209)\end{array}$ & - & $\begin{array}{c}1.799 \\
(0.675-4.791)\end{array}$ & - & - & - & $\begin{array}{c}0.455 \\
(-3.933-4.823)\end{array}$ & - \\
\hline Cancer & - & $\begin{array}{c}0.985 \\
(0.435-2.232)\end{array}$ & - & $\begin{array}{c}0.670 \\
(0.286-1.569)\end{array}$ & - & $\begin{array}{c}1.188 \\
(0.406-3.478)\end{array}$ & - & $\begin{array}{c}-1.052 \\
(-4.229-2.126)\end{array}$ & - \\
\hline $\begin{array}{l}\text { Neurologic } \\
\text { Diseases }\end{array}$ & - & $\begin{array}{c}3.056 \\
(1.639-5.698) \\
*\end{array}$ & $\begin{array}{c}0.843 \\
(0.197-3.617)\end{array}$ & $\begin{array}{c}1.714 \\
(0.909-3.230)\end{array}$ & - & $\begin{array}{c}4.075 \\
(2.028-8.188)^{*}\end{array}$ & $\begin{array}{c}0.733 \\
(0.144-3.732)\end{array}$ & $\begin{array}{c}2.074 \\
(-0.726-4.875)\end{array}$ & - \\
\hline
\end{tabular}




\begin{tabular}{|c|c|c|c|c|c|c|c|c|c|}
\hline & & COVID-19 I & lated Mortality & ICU Adı & mission & Intub & tion & Length of $H$ & ospital Stay \\
\hline Variable & Response & $\begin{array}{c}\text { COR }(95 \% \\
\text { CI })\end{array}$ & $\operatorname{AOR}(95 \%$ CI) & $\begin{array}{c}\text { COR }(95 \% \\
\text { CI) }\end{array}$ & $\begin{array}{l}\text { AOR }(95 \% \\
\text { CI })\end{array}$ & COR (95\% CI) & $\operatorname{AOR}(95 \%$ CI) & CB $(95 \% \mathrm{CI})$ & AB (95\% CI) \\
\hline Heart Failure & - & $\begin{array}{c}4.191 \\
(2.423-7.249) \\
*\end{array}$ & $\begin{array}{c}0.956 \\
(0.298-3.065)\end{array}$ & $\begin{array}{c}4.239 \\
(2.452-7.330) \\
*\end{array}$ & $\begin{array}{c}7.894 \\
(1.391- \\
44.818)^{*}\end{array}$ & $\begin{array}{c}0.754 \\
(0.344-1.651)\end{array}$ & - & $\begin{array}{c}1.372 \\
(-1.048-3.793)\end{array}$ & - \\
\hline $\begin{array}{c}\text { Autoimmune } \\
\text { Diseases }\end{array}$ & - & $\begin{array}{c}1.462 \\
(0.755-2.832)\end{array}$ & - & $\begin{array}{c}0.755 \\
(0.365-1.562)\end{array}$ & - & $\begin{array}{c}0.604 \\
(0.182-1.999)\end{array}$ & - & $\begin{array}{c}1.491 \\
(-1.342-4.325)\end{array}$ & - \\
\hline $\begin{array}{c}\text { Other Respiratory } \\
\text { Disease }\end{array}$ & - & $\begin{array}{c}1.154 \\
(0.669-1.990)\end{array}$ & - & $\begin{array}{c}1.127 \\
(0.664-1.914)\end{array}$ & - & $\begin{array}{c}1.035 \\
(0.477-2.245)\end{array}$ & - & $\begin{array}{c}-.681 \\
(-2.938-1.576)\end{array}$ & - \\
\hline $\begin{array}{c}\text { Other } \\
\text { Cardiovascular } \\
\text { Diseases }\end{array}$ & - & $\begin{array}{c}1.281 \\
(0.576-2.849)\end{array}$ & - & $\begin{array}{c}3.052 \\
(1.461-6.372) \\
*\end{array}$ & $\begin{array}{c}0.340 \\
(0.018- \\
6.556)\end{array}$ & $\begin{array}{c}1.283 \\
(0.436-3.776)\end{array}$ & - & $\begin{array}{c}-.551 \\
(-3.877-2.775)\end{array}$ & - \\
\hline Pregnancy & - & - & - & - & - & - & - & $\begin{array}{c}-6.875 \\
(-13.632-- \\
0.119)^{*}\end{array}$ & - \\
\hline Documented Fever & - & $\begin{array}{c}1.132 \\
(0.765-1.675)\end{array}$ & - & $\begin{array}{c}1.096 \\
(0.750-1.600)\end{array}$ & - & $\begin{array}{c}1.163 \\
(0.679-1.993)\end{array}$ & - & $\begin{array}{c}0.724 \\
(-0.848-2.296)\end{array}$ & - \\
\hline \multirow[b]{2}{*}{ Chest X-ray } & Clear & $\mathrm{R}$ & $\mathrm{R}$ & $\mathrm{R}$ & $\mathrm{R}$ & $\mathrm{R}$ & $\mathrm{R}$ & \multirow{2}{*}{$\begin{array}{c}1.129 \\
(0.140-2.118)^{*}\end{array}$} & \multirow{2}{*}{$\begin{array}{c}0.631 \\
(-1.075-2.337)\end{array}$} \\
\hline & Unilateral & $\begin{array}{c}3.422 \\
(1.489-7.866) \\
*\end{array}$ & $\begin{array}{c}3.995 \\
(0.744-21.456)\end{array}$ & 2.884 & $\begin{array}{c}2.245 \\
(0.165- \\
30.617)\end{array}$ & $\begin{array}{c}4.558 \\
(1.297-16.016)^{*}\end{array}$ & $\begin{array}{c}6.307 \\
(0.766-51.925)\end{array}$ & & \\
\hline
\end{tabular}




\begin{tabular}{|c|c|c|c|c|c|c|c|c|c|}
\hline \multirow[b]{2}{*}{ Variable } & \multirow[b]{2}{*}{ Response } & \multicolumn{2}{|c|}{ COVID-19 Related Mortality } & \multicolumn{2}{|c|}{ ICU Admission } & \multicolumn{2}{|c|}{ Intubation } & \multicolumn{2}{|c|}{ Length of Hospital Stay } \\
\hline & & $\begin{array}{c}\text { COR }(95 \% \\
\text { CI })\end{array}$ & $\operatorname{AOR}(95 \% \mathrm{CI})$ & $\begin{array}{c}\operatorname{COR}(95 \% \\
\text { CI })\end{array}$ & $\begin{array}{c}\text { AOR }(95 \% \\
\text { CI })\end{array}$ & $\operatorname{COR}(95 \% \mathrm{CI})$ & $\operatorname{AOR}(95 \% \mathrm{CI})$ & CB $(95 \%$ CI) & $\mathrm{AB}(95 \% \mathrm{CI})$ \\
\hline & & & & $\begin{array}{c}(1.295-6.423) \\
*\end{array}$ & & & & & \\
\hline & Bilateral & $\begin{array}{c}3.454 \\
(1.621-7.360) \\
*\end{array}$ & $\begin{array}{c}2.187 \\
(0.488-9.797)\end{array}$ & $\begin{array}{c}3.554 \\
(1.730-7.300) \\
*\end{array}$ & $\begin{array}{c}1.465 \\
(0.126- \\
16.988)\end{array}$ & $\begin{array}{c}3.375 \\
(1.031-11.048)^{*}\end{array}$ & $\begin{array}{c}1.820 \\
(0.259-12.790)\end{array}$ & & \\
\hline \multirow{2}{*}{$\begin{array}{l}\text { Computerized } \\
\text { Tomographic } \\
\text { Pulmonary } \\
\text { Angiography }\end{array}$} & Negative & $\mathrm{R}$ & $\mathrm{R}$ & $\mathrm{R}$ & $\mathrm{R}$ & $\mathrm{R}$ & $\mathrm{R}$ & $\mathrm{R}$ & $\mathrm{R}$ \\
\hline & Positive & $\begin{array}{c}0.540 \\
(0.056-5.208)\end{array}$ & - & $\begin{array}{c}1.350 \\
(0.213-8.551)\end{array}$ & - & - & - & $\begin{array}{c}-10.207 \\
(-21.663-1.248)\end{array}$ & - \\
\hline IL-6 & - & $\begin{array}{c}1.004 \\
(0.993-1.014)\end{array}$ & - & $\begin{array}{c}1.000 \\
(0.989-1.010)\end{array}$ & - & $\begin{array}{c}1.006 \\
(0.995-1.017)\end{array}$ & - & $\begin{array}{c}-0.023 \\
(-0.063-0.016)\end{array}$ & - \\
\hline $\mathrm{BNP}$ & - & $\begin{array}{c}1.001 \\
(1.000-1.001)\end{array}$ & - & $\begin{array}{c}1.000 \\
(1.000-1.001)\end{array}$ & - & $\begin{array}{c}1.000 \\
(1.000-1.001)\end{array}$ & - & - & - \\
\hline Troponin & - & $\begin{array}{c}4.641 \\
(2.917-7.383) \\
*\end{array}$ & $\begin{array}{c}3.060 \\
(1.156-8.102)^{*}\end{array}$ & $\begin{array}{c}3.384 \\
(2.139-5.352) \\
*\end{array}$ & $\begin{array}{c}0.655 \\
(0.118- \\
3.620)\end{array}$ & $\begin{array}{c}2.974 \\
(1.693-5.223)^{*}\end{array}$ & $\begin{array}{c}1.544 \\
(0.563-4.234)\end{array}$ & $\begin{array}{c}-0.607 \\
(-2.708-1.494)\end{array}$ & - \\
\hline Procalcitonin & - & $\begin{array}{c}1.036 \\
(0.989-1.085)\end{array}$ & - & $\begin{array}{c}1.013 \\
(0.971-1.058)\end{array}$ & - & $\begin{array}{c}1.034 \\
(0.989-1.081)\end{array}$ & - & $\begin{array}{c}0.198 \\
(-0.004-0.400)\end{array}$ & - \\
\hline Hemoglobin & - & 0.788 & $\begin{array}{c}0.896 \\
(0.759-1.058)\end{array}$ & 0.853 & 0.771 & $\begin{array}{c}0.812 \\
(0.733-0.899)\end{array}$ & $\begin{array}{c}0.917 \\
(0.752-1.118)\end{array}$ & $\begin{array}{c}-0.318 \\
(-0.618--0.019)\end{array}$ & $\begin{array}{c}0.164 \\
(-0.323-0.651)\end{array}$ \\
\hline
\end{tabular}




\begin{tabular}{|c|c|c|c|c|c|c|c|c|c|}
\hline \multirow[b]{2}{*}{ Variable } & \multirow[b]{2}{*}{ Response } & \multicolumn{2}{|c|}{ COVID-19 Related Mortality } & \multicolumn{2}{|c|}{ ICU Admission } & \multicolumn{2}{|c|}{ Intubation } & \multicolumn{2}{|c|}{ Length of Hospital Stay } \\
\hline & & $\begin{array}{c}\operatorname{COR}(95 \% \\
\text { CI })\end{array}$ & $\operatorname{AOR}(95 \%$ CI) & $\begin{array}{c}\text { COR }(95 \% \\
\text { CI })\end{array}$ & $\begin{array}{c}\text { AOR }(95 \% \\
\text { CI) }\end{array}$ & COR $(95 \%$ CI) & $\operatorname{AOR}(95 \% \mathrm{CI})$ & CB $(95 \% \mathrm{CI})$ & AB $(95 \% \mathrm{CI})$ \\
\hline & & $\begin{array}{c}(0.728-0.852) \\
*\end{array}$ & & $\begin{array}{c}(0.792-0.918) \\
*\end{array}$ & $\begin{array}{l}(0.554- \\
1.072)\end{array}$ & & & & \\
\hline White Blood Cells & - & $\begin{array}{c}1.035 \\
(1.009-1.062) \\
*\end{array}$ & $\begin{array}{c}0.835 \\
(0.613-1.138)\end{array}$ & $\begin{array}{c}1.013 \\
(0.996-1.030)\end{array}$ & - & $\begin{array}{c}1.001 \\
(0.997-1.005)\end{array}$ & - & $\begin{array}{c}0.002 \\
(-0.014-0.019)\end{array}$ & - \\
\hline Neutrophils & - & $\begin{array}{c}1.125 \\
(1.084-1.167) \\
*\end{array}$ & $\begin{array}{c}1.306 \\
(0.933-1.828)\end{array}$ & $\begin{array}{c}1.058 \\
(1.025-1.092) \\
*\end{array}$ & $\begin{array}{l}1.003 \\
(0.984- \\
1.023)\end{array}$ & $\begin{array}{c}1.002 \\
(0.997-1.006)\end{array}$ & - & $\begin{array}{c}0.004 \\
(-0.014-0.023)\end{array}$ & - \\
\hline Lymphocyte & - & $\begin{array}{c}1.013 \\
(0.971-1.058)\end{array}$ & - & $\begin{array}{c}1.011 \\
(0.969-1.055)\end{array}$ & - & $\begin{array}{c}0.931 \\
(0.769-1.128)\end{array}$ & - & $\begin{array}{c}-0.051 \\
(-0.241-0.139)\end{array}$ & - \\
\hline Platelet & - & $\begin{array}{c}1.000 \\
(1.000-1.001)\end{array}$ & - & $\begin{array}{c}1.000 \\
(1.000-1.001)\end{array}$ & - & $\begin{array}{c}0.999 \\
(0.997-1.000)\end{array}$ & - & $\begin{array}{c}0.003 \\
(0.000-0.005)\end{array}$ & - \\
\hline CRP & - & $\begin{array}{c}1.000 \\
(1.000-1.000)\end{array}$ & - & $\begin{array}{c}1.000 \\
(0.999-1.000)\end{array}$ & - & $\begin{array}{c}1.000 \\
(1.000-1.000)\end{array}$ & - & $\begin{array}{c}0.001 \\
(0.000-0.002)\end{array}$ & - \\
\hline Ferritin & - & $\begin{array}{c}1.001 \\
(1.000-1.001)\end{array}$ & - & $\begin{array}{c}1.001 \\
(1.000-1.001)\end{array}$ & - & $\begin{array}{c}1.001 \\
(1.000-1.001)\end{array}$ & - & $\begin{array}{c}0.002 \\
(0.001-0.004)\end{array}$ & - \\
\hline D-dimer & - & $\begin{array}{c}3.680 \\
(1.803-7.509) \\
*\end{array}$ & $\begin{array}{c}0.945 \\
(0.270-3.305)\end{array}$ & $\begin{array}{c}2.274 \\
(1.268-4.077) \\
*\end{array}$ & $\begin{array}{l}1.907 \\
(0.087- \\
41.862)\end{array}$ & $\begin{array}{c}2.951 \\
(1.048-8.310)^{*}\end{array}$ & $\begin{array}{c}2.766 \\
(0.310-24.703)\end{array}$ & $\begin{array}{c}2.398 \\
(0.543-4.254)^{*}\end{array}$ & $\begin{array}{c}0.743 \\
(-2.210-3.697)\end{array}$ \\
\hline $\mathrm{LDH}$ & - & 1.001 & $\begin{array}{c}1.002 \\
(1.001-1.003)^{*}\end{array}$ & $\begin{array}{c}1.001 \\
(1.000-1.001)\end{array}$ & - & $\begin{array}{c}1.001 \\
(1.001-1.002)^{*}\end{array}$ & $\begin{array}{c}1.001 \\
(1.000-1.002)\end{array}$ & $\begin{array}{c}9.588 \mathrm{E}-5 \\
(-0.001-0.002)\end{array}$ & - \\
\hline
\end{tabular}




\begin{tabular}{|c|c|c|c|c|c|c|c|c|c|}
\hline \multirow[b]{2}{*}{ Variable } & \multirow[b]{2}{*}{ Response } & \multicolumn{2}{|c|}{ COVID-19 Related Mortality } & \multicolumn{2}{|c|}{ ICU Admission } & \multicolumn{2}{|c|}{ Intubation } & \multicolumn{2}{|c|}{ Length of Hospital Stay } \\
\hline & & $\begin{array}{c}\text { COR }(95 \% \\
\text { CI })\end{array}$ & $\operatorname{AOR}(95 \% \mathrm{CI})$ & $\begin{array}{c}\text { COR }(95 \% \\
\text { CI })\end{array}$ & $\begin{array}{c}\operatorname{AOR}(95 \% \\
\text { CI })\end{array}$ & $\operatorname{COR}(95 \% \mathrm{CI})$ & $\operatorname{AOR}(95 \% \mathrm{CI})$ & CB $(95 \% \mathrm{CI})$ & $\mathrm{AB}(95 \% \mathrm{CI})$ \\
\hline & & $\begin{array}{c}(1.001-1.002) \\
*\end{array}$ & & & & & & & \\
\hline Creatinine & - & $\begin{array}{c}3.756 \\
(2.640-5.343) \\
*\end{array}$ & $\begin{array}{c}1.128 \\
(0.448-2.840)\end{array}$ & $\begin{array}{c}2.327 \\
(1.657-3.268) \\
*\end{array}$ & $\begin{array}{c}0.901 \\
(0.176- \\
4.608)\end{array}$ & $\begin{array}{c}2.306 \\
(1.440-3.693)^{*}\end{array}$ & $\begin{array}{c}1.856 \\
(0.643-5.357)\end{array}$ & $\begin{array}{c}-0.710 \\
(-2.149-0.730)\end{array}$ & - \\
\hline Potassium & - & $\begin{array}{c}1.008 \\
(1.002-1.014) \\
*\end{array}$ & $\begin{array}{c}0.986 \\
(0.922-1.056)\end{array}$ & $\begin{array}{c}1.013 \\
(1.006-1.019) \\
*\end{array}$ & $\begin{array}{c}1.051 \\
(0.951- \\
1.161)\end{array}$ & $\begin{array}{c}0.926 \\
(0.843-1.017)\end{array}$ & - & $\begin{array}{c}0.023 \\
(-0.001-0.048)\end{array}$ & - \\
\hline Sodium & - & $\begin{array}{c}1.001 \\
(0.995-1.006)\end{array}$ & - & $\begin{array}{c}0.992 \\
(0.987-0.997) \\
*\end{array}$ & $\begin{array}{c}1.024 \\
(0.932- \\
1.125)\end{array}$ & $\begin{array}{c}1.027 \\
(1.007-1.047)^{*}\end{array}$ & $\begin{array}{c}1.046 \\
(0.992-1.103)\end{array}$ & $\begin{array}{c}-0.022 \\
(-0.044-0.000)\end{array}$ & - \\
\hline Urea & - & $\begin{array}{c}1.000 \\
(1.000-1.000)\end{array}$ & - & $\begin{array}{c}1.000 \\
(1.000-1.000)\end{array}$ & - & $\begin{array}{c}1.000 \\
(1.000-1.000)\end{array}$ & - & $\begin{array}{c}0.000 \\
(-0.001-0.000)\end{array}$ & - \\
\hline ALT & - & $\begin{array}{c}1.000 \\
(0.999-1.002)\end{array}$ & - & $\begin{array}{c}1.001 \\
(0.999-1.003)\end{array}$ & - & $\begin{array}{c}1.004 \\
(1.002-1.007)^{*}\end{array}$ & $\begin{array}{c}1.005 \\
(0.996-1.014)\end{array}$ & $\begin{array}{c}-0.005 \\
(-0.011-0.002)\end{array}$ & - \\
\hline AST & - & $\begin{array}{c}1.002 \\
(1.000-1.003)\end{array}$ & - & $\begin{array}{c}1.000 \\
(0.999-1.001)\end{array}$ & - & $\begin{array}{c}1.003 \\
(1.001-1.005)^{*}\end{array}$ & $\begin{array}{c}1.001 \\
(0.999-1.003)\end{array}$ & $\begin{array}{c}-0.001 \\
(-0.006-0.003)\end{array}$ & - \\
\hline Bilirubin & - & $\begin{array}{c}1.222 \\
(1.058-1.410) \\
*\end{array}$ & $\begin{array}{c}0.837 \\
(0.450-1.555)\end{array}$ & $\begin{array}{c}1.093 \\
(0.959-1.245)\end{array}$ & - & $\begin{array}{c}1.090 \\
(0.939-1.264)\end{array}$ & - & $\begin{array}{c}0.662 \\
(0.069-1.254)^{*}\end{array}$ & $\begin{array}{c}0.138 \\
(-0.752-1.029)\end{array}$ \\
\hline
\end{tabular}




\begin{tabular}{|c|c|c|c|c|c|c|c|c|c|}
\hline & & \multicolumn{2}{|c|}{ COVID-19 Related Mortality } & \multicolumn{2}{|c|}{ ICU Admission } & \multicolumn{2}{|c|}{ Intubation } & \multicolumn{2}{|c|}{ Length of Hospital Stay } \\
\hline Variable & Response & $\begin{array}{c}\text { COR }(95 \% \\
\text { CI })\end{array}$ & $\operatorname{AOR}(95 \% \mathrm{CI})$ & $\begin{array}{c}\text { COR }(95 \% \\
\text { CI) }\end{array}$ & $\begin{array}{c}\text { AOR (95\% } \\
\text { CI) }\end{array}$ & $\operatorname{COR}(95 \% \mathrm{CI})$ & $\operatorname{AOR}(95 \% \mathrm{CI})$ & CB $(95 \%$ CI $)$ & $\mathrm{AB}(95 \% \mathrm{CI})$ \\
\hline Direct Bilirubin & - & $\begin{array}{c}1.001 \\
(0.995-1.008)\end{array}$ & - & $\begin{array}{c}1.006 \\
(1.000-1.013)\end{array}$ & - & $\begin{array}{c}0.954 \\
(0.900-1.011)\end{array}$ & - & $\begin{array}{c}-0.006 \\
(-0.035-0.022)\end{array}$ & - \\
\hline Last HBA1C & - & $\begin{array}{c}2.146 \\
(0.886-5.197)\end{array}$ & - & $\begin{array}{c}0.987 \\
(0.349-2.788)\end{array}$ & - & $\begin{array}{c}0.764 \\
(0.167-3.495)\end{array}$ & - & $\begin{array}{c}0.692 \\
(-3.749-5.134)\end{array}$ & - \\
\hline \multirow{3}{*}{ O2 Saturation } & $=>94$ & $\mathrm{R}$ & $\mathrm{R}$ & $\mathrm{R}$ & $\mathrm{R}$ & $\mathrm{R}$ & $\mathrm{R}$ & \multirow{3}{*}{$\begin{array}{c}1.471 \\
(0.705-2.236)^{*}\end{array}$} & \multirow{3}{*}{$\begin{array}{c}0.215 \\
(-1.037-1.466)\end{array}$} \\
\hline & $90-94$ & $\begin{array}{c}1.186 \\
(0.708-1.986)\end{array}$ & $\begin{array}{c}1.558 \\
(0.515-4.711)\end{array}$ & $\begin{array}{c}1.023 \\
(0.616-1.698)\end{array}$ & $\begin{array}{c}1.020 \\
(0.141- \\
7.397)\end{array}$ & $\begin{array}{c}1.666 \\
(0.750-3.697)\end{array}$ & $\begin{array}{c}5.536 \\
(0.771-39.750)\end{array}$ & & \\
\hline & $<90$ & $\begin{array}{c}2.630 \\
(1.729-3.998) \\
*\end{array}$ & $\begin{array}{c}2.761 \\
(1.066-7.155)^{*}\end{array}$ & $\begin{array}{c}2.945 \\
(1.965-4.412) \\
*\end{array}$ & $\begin{array}{c}0.938 \\
(0.162- \\
5.417)\end{array}$ & $\begin{array}{c}4.015 \\
(2.089-7.715)^{*}\end{array}$ & $\begin{array}{c}16.585 \\
(2.892-95.118)^{*}\end{array}$ & & \\
\hline Steroids & - & $\begin{array}{c}1.609 \\
(0.896-2.890)\end{array}$ & - & $\begin{array}{c}0.782 \\
(0.471-1.300)\end{array}$ & - & $\begin{array}{c}5.533 \\
(1.334-22.947)^{*}\end{array}$ & $\begin{array}{c}0.551 \\
(0.077-3.940)\end{array}$ & $\begin{array}{c}1.837 \\
(-0.335-4.010)\end{array}$ & - \\
\hline \multirow{2}{*}{ Vaccination status } & No & $\mathrm{R}$ & $\mathrm{R}$ & $\mathrm{R}$ & $\mathrm{R}$ & $\mathrm{R}$ & $\mathrm{R}$ & $\mathrm{R}$ & $\mathrm{R}$ \\
\hline & Yes & $\begin{array}{c}0.582 \\
(0.196-1.726)\end{array}$ & - & $\begin{array}{c}1.044 \\
(0.426-2.555)\end{array}$ & - & $\begin{array}{c}0.351 \\
(0.047-2.631)\end{array}$ & - & $\begin{array}{c}0.764 \\
(-2.939-4.466)\end{array}$ & - \\
\hline Tocilizumab & - & $\begin{array}{c}1.584 \\
(0.930-2.698)\end{array}$ & - & 2.479 & 1.675 & $\begin{array}{c}1.483 \\
(0.725-3.032)\end{array}$ & - & $\begin{array}{c}2.815 \\
(0.553-5.078)^{*}\end{array}$ & $\begin{array}{c}2.689 \\
(-0.757-6.134)\end{array}$ \\
\hline
\end{tabular}




\begin{tabular}{|c|c|c|c|c|c|c|c|c|c|}
\hline \multirow[b]{2}{*}{ Variable } & \multirow[b]{2}{*}{ Response } & \multicolumn{2}{|c|}{ COVID-19 Related Mortality } & \multicolumn{2}{|c|}{ ICU Admission } & \multicolumn{2}{|c|}{ Intubation } & \multicolumn{2}{|c|}{ Length of Hospital Stay } \\
\hline & & $\begin{array}{c}\text { COR }(95 \% \\
\text { CI) }\end{array}$ & $\operatorname{AOR}(95 \%$ CI) & $\begin{array}{c}\text { COR }(95 \% \\
\text { CI) }\end{array}$ & $\begin{array}{c}\text { AOR }(95 \% \\
\text { CI) }\end{array}$ & COR $(95 \% \mathrm{CI})$ & AOR $(95 \% \mathrm{CI})$ & CB $(95 \%$ CI $)$ & $\mathrm{AB}(\mathbf{9 5 \%} \mathrm{CI})$ \\
\hline & & & & $\begin{array}{c}(1.494-4.114) \\
*\end{array}$ & $\begin{array}{l}(0.294- \\
9.524)\end{array}$ & & & & \\
\hline Remedisvir & - & $\begin{array}{c}0.916 \\
(0.621-1.352)\end{array}$ & - & $\begin{array}{c}0.591 \\
(0.414-0.845) \\
*\end{array}$ & $\begin{array}{c}0.192 \\
(0.044- \\
0.835)^{*}\end{array}$ & $\begin{array}{c}1.094 \\
(0.645-1.856)\end{array}$ & - & $\begin{array}{c}1.564 \\
(0.043-3.085)^{*}\end{array}$ & $\begin{array}{c}0.054 \\
(-2.380-2.488)\end{array}$ \\
\hline
\end{tabular}

"P-value $<0.05$, R: Reference Group, COR: Crude Odds Ratio, AOR: Adjusted Odds Ratio, CB: Crude B Coefficient, AB: Adjusted B Coefficient.

\section{Comparison between the First and the Second Waves of the Pandemic}

The analysis that compared between the first and the second waves in COVID-19 outcomes showed that there was no significant

difference between the waves in regards to COVID-19 related mortality, intubation or length of hospital stay. Regarding COVID-19 related mortality, $17.7 \%$ of the patients died in the first wave while $25.8 \%$ of the patients died in the second wave. However, the second wave had significantly higher rates of ICU admission compared to the first one (Table 4; AOR=4.728; 95\% CI: 3.079-7.259) with $11.3 \%$ ICU admission in the first wave and 37.6\% ICU admission in the second wave. Furthermore, after adjusting for patients' demographics and comorbidities, the second wave also had higher rates of ICU admissions compared to the first wave (Table 4; 
$\mathrm{AOR}=5.466 ; 95 \% \mathrm{CI}: 3.402-8.781)$. In addition, the model that was adjusted for patients' demographics, comorbidities, steroids use in the treatment and vaccination status also showed that the second wave had higher rates of ICU admissions compared to the first

wave (Table 4; AOR=4.797; 95\% CI: 2.903-7.927).

Table 4. Regression Analysis for the Comparison between the First and the Second Wave of the Pandemic. Add Percentages.

\begin{tabular}{|c|c|c|c|c|c|c|c|c|c|c|}
\hline & $\begin{array}{c}\text { COVID-19 } \\
\text { Related } \\
\text { Mortality }\end{array}$ & $\begin{array}{c}\text { Mortality } \\
\text { Wave } 1\end{array}$ & $\begin{array}{c}\text { Mortality } \\
\text { Wave } 2\end{array}$ & ICU Admission & $\begin{array}{c}\text { ICU } \\
\text { Admission } \\
\text { Wave } 1\end{array}$ & $\begin{array}{c}\text { ICU } \\
\text { Admission } \\
\text { Wave } 2\end{array}$ & Intubation & $\begin{array}{c}\text { Intubation } \\
\text { Wave } 1\end{array}$ & $\begin{array}{c}\text { Intubation } \\
\text { Wave } 2\end{array}$ & $\begin{array}{c}\text { Length of } \\
\text { Hospital Stay }\end{array}$ \\
\hline Variable & OR $(95 \%$ CI $)$ & $\begin{array}{c}n \\
(\%)\end{array}$ & $\begin{array}{c}n \\
(\%)\end{array}$ & OR (95\% CI) & $\begin{array}{c}n \\
(\%)\end{array}$ & $\begin{array}{c}n \\
(\%)\end{array}$ & OR $(95 \%$ CI $)$ & $\begin{array}{c}n \\
(\%)\end{array}$ & $\begin{array}{c}\mathbf{n} \\
(\%)\end{array}$ & OR $(95 \%$ CI) \\
\hline $\begin{array}{l}\text { Waves (Not } \\
\text { Adjusted) }\end{array}$ & $\begin{array}{c}1.432 \\
(0.997-2.055)\end{array}$ & \multirow{3}{*}{$\begin{array}{c}47 \\
(17.7 \%)\end{array}$} & \multirow{3}{*}{$\begin{array}{c}124 \\
(25.8)\end{array}$} & $\begin{array}{c}4.728 \\
(3.079-7.259)^{*}\end{array}$ & \multirow{3}{*}{$\begin{array}{c}29 \\
(11.3 \%)\end{array}$} & \multirow{3}{*}{$\begin{array}{c}178 \\
(37.6)\end{array}$} & $\begin{array}{c}1.041 \\
(0.640-1.692)\end{array}$ & \multirow{3}{*}{$\begin{array}{c}28 \\
(10.6 \%)\end{array}$} & \multirow{3}{*}{$\begin{array}{c}52 \\
(10.9 \%)\end{array}$} & $\begin{array}{c}-0.354 \\
(-1.738-1.031)\end{array}$ \\
\hline $\begin{array}{c}\text { Waves } \\
\text { (Adjusted for } \\
\text { Demographics } \\
\text { and } \\
\text { Comorbidities) }\end{array}$ & $\begin{array}{c}1.351 \\
(0.905-2.018)\end{array}$ & & & $\begin{array}{c}5.466 \\
(3.402-8.781)^{*}\end{array}$ & & & $\begin{array}{c}0.980 \\
(0.584-1.644)\end{array}$ & & & $\begin{array}{c}-0.549 \\
(-1.980-0.881)\end{array}$ \\
\hline $\begin{array}{c}\text { Waves } \\
\text { (Adjusted for } \\
\text { Demographics, } \\
\text { Comorbidities, } \\
\text { Steroids use } \\
\text { and }\end{array}$ & $\begin{array}{c}1.402 \\
(0.904-2.176)\end{array}$ & & & $\begin{array}{c}4.797 \\
(2.903-7.927)^{*}\end{array}$ & & & $\begin{array}{c}0.939 \\
(0.524-1.683)\end{array}$ & & & $\begin{array}{c}-1.296 \\
(-2.887-0.295)\end{array}$ \\
\hline
\end{tabular}




\begin{tabular}{|c|c|c|c|c|c|c|c|c|c|c|}
\hline & $\begin{array}{l}\text { COVID-19 } \\
\text { Related } \\
\text { Mortality }\end{array}$ & $\begin{array}{c}\text { Mortality } \\
\text { Wave 1 }\end{array}$ & $\begin{array}{c}\text { Mortality } \\
\text { Wave 2 }\end{array}$ & ICU Admission & $\begin{array}{c}\text { ICU } \\
\text { Admission } \\
\text { Wave } 1\end{array}$ & $\begin{array}{c}\text { ICU } \\
\text { Admission } \\
\text { Wave } 2\end{array}$ & Intubation & $\begin{array}{c}\text { Intubation } \\
\text { Wave } 1\end{array}$ & $\begin{array}{l}\text { Intubation } \\
\text { Wave } 2\end{array}$ & $\begin{array}{l}\text { Length of } \\
\text { Hospital Stay }\end{array}$ \\
\hline Variable & OR (95\% CI) & $\begin{array}{c}\mathrm{n} \\
(\%)\end{array}$ & $\begin{array}{c}n \\
(\%)\end{array}$ & OR (95\% CI) & $\begin{array}{c}n \\
(\%)\end{array}$ & $\begin{array}{c}\mathrm{n} \\
(\%)\end{array}$ & OR $(95 \% \mathrm{CI})$ & $\begin{array}{c}n \\
(\%)\end{array}$ & $\begin{array}{c}\mathrm{n} \\
(\%)\end{array}$ & OR $(95 \% \mathrm{CI})$ \\
\hline $\begin{array}{c}\text { Vaccination } \\
\text { status) }\end{array}$ & & & & & & & & & & \\
\hline
\end{tabular}

\section{Discussion}

According to our knowledge, this is the first study conducted in Jordan and one of few studies conducted in the Middle Eastern region that characterized the clinical features of COVID-19 patients and identified the factors related to COVID-19 severity and mortality.

Our study showed that the most frequent symptoms COVID-19 patients presented with were shortness of breath (35.0\%), cough (15.5\%) and generalized fever (15.0\%). The prevalence of the COVID-19 symptoms varied between the studies as several studies in

Brazil [20], Saudi Arabia [21], Kuwait [16] and China [2] reported higher prevalence of fever and cough with fever being the most 
common symptom among their patients. In addition, we reported a low percentage of asymptomatic patients $(<1 \%)$ whereas studies in the USA [22], Kuwait [16] and China [23] reported that around 40\% of their patients were asymptomatic. However, these differences can be explained by the fact that the majority of these studies were done at the beginning of the pandemic when most of the COVID19 patients regardless of their symptom's severity were admitted to the hospitals. Furthermore, our findings demonstrated that the most common radiological finding in chest X-rays among COVID-19 patients was bilateral infiltrates (70.9\%). Similarly, studies conducted in China [2] and the United Kingdom [24] also reported that the most common finding in chest x-rays among COVID-19 patients was bilateral infiltration. In addition, most of the patients in our study who underwent HRCT imaging had ground glass opacification (70.0\%) which is also consistent with previous studies conducted in China [2]. Moreover, more than 50\% of the patients in our cohort required non-invasive oxygen support systems with the face masks and non-rebreather masks being the most commonly used. Whereas only $10.8 \%$ of the patients in our study needed invasive intubation. The rates of using non-invasive oxygen support systems are similar to what is reported in previous studies [24]. However, the rates of intubation in our cohort were lower compared to previous studies which reported that around $20 \%$ of the admitted patients required invasive intubation [8]. This low rate of intubation might be a consequence of refusal of intubation by the patients or the patients' relatives due to insufficient knowledge regarding COVID-19 among the Jordanian population [25]. Moreover, mortality rates in our cohort were $23 \%$ which is quite similar to the rates reported in previous studies conducted in Kuwait [16], Spain [26] and Germany [27]. In addition, the ICU mortality rates in our study 
(53.1\%) are consistent with several studies conducted in Italy [28], Germany [27], Saudi Arabia [21] and Kuwait [16]. However, our study had high rates of comorbidities as almost half of our study cohort had diabetes and hypertension.

Regarding the predictors of COVID-19 related mortality and severity, CKD, positive troponin, LDH and O2 saturation below $90 \%$ were significantly associated with higher risk for COVID-19 mortality. Previous studies showed that positive troponin was a significant predictor of COVID-19 mortality indicating that cardiovascular injury was associated with higher mortality among COVID-19 patients [29]. However, in contrast to our study, studies showed that another cardiac marker, BNP was also associated with higher risk for mortality [30]. Additionally, LDH was significantly associated with higher risk of mortality which was consistent with previous studies that showed that higher LDH levels were independent predictors for mortality [29]. LDH is an enzyme that is found in many organs with its elevation indicating multiorgan injuries [31]. Although in our study diabetes and hypertension were not predictors of mortality which contradict the results of several studies [16], our study showed that CKD, which is a common complication of hypertension and diabetes was a major predictor of COVID-19 mortality. Similar to our study, several articles showed that low $\mathrm{O} 2$ saturation at presentation was associated with higher COVID-19 mortality as low O2 saturation is associated with higher degree of lung injury and more delayed presentation [32]. Also, our analysis showed that low O2 saturation was a significant predictor of invasive intubation. Surprisingly, the results revealed that the presentation with GI symptoms was associated with lower risk of invasive intubation. A meta-analysis showed that the prevalence of GI symptoms among COVID-19 patients was $20 \%$ and it 
was not associated with higher risk for mortality [33]. In addition, a cohort study showed that patients who presented with GI symptoms had a significantly lower COVID-19 severity [34]. Furthermore, our analysis revealed that heart failure and the use of remdesivir in treating patients were the only significant factors associated with ICU admission. Studies showed that 1 in 4 patients with heart failure hospitalized for COVID-19 infection died [35]. Moreover, consistent with our findings, cohort studies showed that remdesivir reduced COVID-19 ICU admission but not mortality [36, 37].

It is important to mention that in our study age and sex were not significantly associated with higher COVID-19 severity or mortality. The majority of the studies in the literature showed that older age is a significant predictor of COVID-19 severity and mortality [38]. However, our findings can be explained by the fact that half of our patients were above 65 with a mean age of 63.15 for the whole cohort which limits the comparison in COVID-19 outcomes between elderly and young patients. On the other hand, the results about the association between COVID-19 mortality and sex were inconsistent in the literature with some studies reporting a higher risk of mortality among male sex [39] and others reporting a lack of a significant difference between males and females in the rates of COVID-19 mortality [40]. Additionally, our study showed that vaccination status was not a significant predictor of COVID-19 mortality or severity which might be explained by the fact that at the time of conducting this study COVID-19 vaccines were not widely available as only $3.2 \%$ of our study cohort were vaccinated. Despite the fact that several studies showed that steroids (dexamethasone and methylprednisolone) use among COVID-19 patients reduced COVID-19 severity and mortality [41], our study 
showed no such association. Yet, in our study, steroids were used to treat a very high percentage of patients $(88.6 \%)$ which limits the comparison in the outcomes between patients who were and were not treated with steroids.

Our study showed that the second wave of the pandemic was associated with higher rate of ICU admissions but not intubation or mortality compared to the first wave. Even after adjusting for patients' characteristics, vaccination status and methods of treatment, the second wave had significantly higher rates of ICU admissions. These findings can be explained by the fact that the second wave was driven by the transmission of the delta and alpha variants which might indicate that these variants were associated with higher disease severity compared to the original variant that spread in Wuhan. Studies showed that the Alpha variant was significantly associated with higher rates of mortality in multiple European countries [42, 43]. However, none of them found that it was also associated with higher ICU admission rates.

However, this study has several limitations. First, this was a single center cohort study which limits the generalizability of its findings. Second, although our analysis was adjusted for confounding variables, the risk of confounding bias cannot be totally excluded. Third, we did not perform genetic sequencing to prove the SARS-COV-2 variants that affected the patients during the two waves of the pandemic which might limit the interpretations of our analysis that compared the 2 waves disease severity and mortality.

\section{Conclusion}


To conclude, we provide the detailed clinical characteristics, investigations and treatment methods of 745 COVID-19 patients. The overall rates of COVID-19 related mortality, ICU admission and invasive intubation were $23.0 \%, 28.3 \%$ and $10.8 \%$, respectively. Our study showed that CKD, positive troponin, $\mathrm{LDH}$ and $\mathrm{O} 2$ saturation $<90 \%$ at admission were significant predictors of COVID-19 morality. Moreover, heart failure patients had a significantly higher risk for ICU admission while remdesivir use in treating COVID19 patients was associated with a reduction in the risk of ICU admission. Patients who had O2 saturation $<90 \%$ at admission had a significantly higher risk for invasive intubation whereas patients presenting with GI symptoms had a significantly lower risk for it. Additionally, the second wave of the pandemic had significantly higher rates of ICU admission but not mortality or invasive intubation compared to the first wave.

\section{Contributions}

KA and ASA were involved in Conceptualization; KA, ASA, AAT, MKR, MMH, AHA, DLA, MR and NO were involved in Data curation, Formal analysis, Investigation, Methodology, Project administration, Resources, Software, Validation, Visualization, and Writing the original draft; ASA, KA, AAT and NO were involved in Supervision and Reviewing \& Editing the manuscript.

\section{Data Sharing}

The data associated with this manuscript are available in the Supplementary Material.

\section{Funding}


The author(s) received no financial support for the research, authorship, and/or publication of this article.

\section{Acknowledgments}

None.

\section{References}

1. The species Severe acute respiratory syndrome-related coronavirus: classifying 2019-nCoV and naming it SARS-CoV-2. Nature microbiology. 2020;5(4):536-44. Epub 2020/03/04. doi: 10.1038/s41564-020-0695-z. PubMed PMID: 32123347; PubMed Central PMCID: PMCPMC7095448.

2. Zhu N, Zhang D, Wang W, Li X, Yang B, Song J, et al. A Novel Coronavirus from Patients with Pneumonia in China, 2019. The New England journal of medicine. 2020;382(8):727-33. Epub 2020/01/25. doi: 10.1056/NEJMoa2001017. PubMed PMID: 31978945; PubMed Central PMCID: PMCPMC7092803.

3. Gomes CJBJol, Sciences H. Report of the WHO-China joint mission on coronavirus disease 2019 (COVID-19). 2020;2(3). 4. $(\mathrm{NIH}) \mathrm{NloH}$. Clinical Spectrum of SARS-CoV-2 Infection 2021 [updated 31/10/2021; cited 2021 28/1/2021]. Available from: https://www.covid19treatmentguidelines.nih.gov/overview/clinical-spectrum/.

5. Meters W. COVID-19 CORONAVIRUS PANDEMIC 2021 [updated 27/1/2021; cited 2021 28/1/2021]. Available from: https://www.worldometers.info/coronavirus/.

6. Li X, Xu S, Yu M, Wang K, Tao Y, Zhou Y, et al. Risk factors for severity and mortality in adult COVID-19 inpatients in Wuhan. The Journal of allergy and clinical immunology. 2020;146(1):110-8. Epub 2020/04/16. doi: 10.1016/j.jaci.2020.04.006. PubMed PMID: 32294485; PubMed Central PMCID: PMCPMC7152876.

7. Parohan M, Yaghoubi S, Seraji A, Javanbakht MH, Sarraf $P$, Djalali M. Risk factors for mortality in patients with Coronavirus disease 2019 (COVID-19) infection: a systematic review and meta-analysis of observational studies. The aging male : the official journal of the International Society for the Study of the Aging Male. 2020;23(5):1416-24. Epub 2020/06/09. doi: 10.1080/13685538.2020.1774748. PubMed PMID: 32508193.

8. Palaiodimos L, Kokkinidis DG, Li W, Karamanis D, Ognibene J, Arora S, et al. Severe obesity, increasing age and male sex are independently associated with worse in-hospital outcomes, and higher in-hospital mortality, in a cohort of patients with COVID-19 
in the Bronx, New York. Metabolism: clinical and experimental. 2020;108:154262. Epub 2020/05/19. doi: 10.1016/j.metabol.2020.154262. PubMed PMID: 32422233; PubMed Central PMCID: PMCPMC7228874.

9. Zhou F, Yu T, Du R, Fan G, Liu Y, Liu Z, et al. Clinical course and risk factors for mortality of adult inpatients with COVID-19 in Wuhan, China: a retrospective cohort study. Lancet (London, England). 2020;395(10229):1054-62. Epub 2020/03/15. doi: 10.1016/s0140-6736(20)30566-3. PubMed PMID: 32171076; PubMed Central PMCID: PMCPMC7270627.

10. Huang C, Wang Y, Li X, Ren L, Zhao J, Hu Y, et al. Clinical features of patients infected with 2019 novel coronavirus in Wuhan, China. Lancet (London, England). 2020;395(10223):497-506. Epub 2020/01/28. doi: 10.1016/s0140-6736(20)30183-5. PubMed PMID: 31986264; PubMed Central PMCID: PMCPMC7159299.

11. Cecconi M, Piovani D, Brunetta E, Aghemo A, Greco M, Ciccarelli M, et al. Early Predictors of Clinical Deterioration in a Cohort of 239 Patients Hospitalized for Covid-19 Infection in Lombardy, Italy. Journal of clinical medicine. 2020;9(5). Epub 2020/05/24. doi: 10.3390/jcm9051548. PubMed PMID: 32443899; PubMed Central PMCID: PMCPMC7290833.

12. Wu Z, McGoogan JM. Characteristics of and Important Lessons From the Coronavirus Disease 2019 (COVID-19) Outbreak in China: Summary of a Report of 72314 Cases From the Chinese Center for Disease Control and Prevention. Jama. 2020;323(13):1239-42. Epub 2020/02/25. doi: 10.1001/jama.2020.2648. PubMed PMID: 32091533.

13. Chen T, Wu D, Chen H, Yan W, Yang D, Chen G, et al. Clinical characteristics of 113 deceased patients with coronavirus disease 2019: retrospective study. BMJ (Clinical research ed). 2020;368:m1091. Epub 2020/03/29. doi: 10.1136/bmj.m1091.

PubMed PMID: 32217556; PubMed Central PMCID: PMCPMC7190011 www.icmje.org/coi disclosure.pdf and declare: support from the Tongji Hospital for Pilot Scheme Project and the Chinese National Thirteenth Five Years Project in Science and Technology, National Commission of Health, People's Republic of China, for the submitted work; no financial relationships with any organisation that might have an interest in the submitted work in the previous three years; no other relationships or activities that could appear to have influenced the submitted work.

14. Huang S, Wang J, Liu F, Liu J, Cao G, Yang C, et al. COVID-19 patients with hypertension have more severe disease: a multicenter retrospective observational study. Hypertension research : official journal of the Japanese Society of Hypertension. 2020;43(8):824-31. Epub 2020/06/03. doi: 10.1038/s41440-020-0485-2. PubMed PMID: 32483311; PubMed Central PMCID: PMCPMC7261650.

15. Guo W, Li M, Dong Y, Zhou H, Zhang Z, Tian C, et al. Diabetes is a risk factor for the progression and prognosis of COVID19. 2020;36(7):e3319. doi: https://doi.org/10.1002/dmrr.3319.

16. Alshukry A, Ali H, Ali Y, Al-Taweel T, Abu-Farha M, AbuBaker J, et al. Clinical characteristics of coronavirus disease 2019 (COVID-19) patients in Kuwait. PloS one. 2020;15(11):e0242768. doi: 10.1371/journal.pone.0242768.

17. Nassar Y, Mokhtar A, Elhadidy A, Elsayed M, Mostafa F, Rady A, et al. Outcomes and risk factors for death in patients with coronavirus disease-2019 (COVID-19) pneumonia admitted to the intensive care units of an Egyptian University Hospital. A retrospective cohort study. Journal of infection and public health. 2021;14(10):1381-8. Epub 2021/07/04. doi: 10.1016/j.jiph.2021.06.012. PubMed PMID: 34215561; PubMed Central PMCID: PMCPMC8233058. 
18. Obermeyer Z, Samra JK, Mullainathan S. Individual differences in normal body temperature: longitudinal big data analysis of patient records. BMJ (Clinical research ed). 2017;359:j5468. Epub 2017/12/15. doi: 10.1136/bmj.j5468. PubMed PMID: 29237616; PubMed Central PMCID: PMCPMC5727437 www.icmje.org/coi disclosure.pdf and declare: no financial relationships with any organizations that might have an interest in the submitted work in the previous three years; no other relationships or activities that could appear to have influenced the submitted work.

19. Ostermann M, Joannidis M. Acute kidney injury 2016: diagnosis and diagnostic workup. Critical care (London, England). 2016;20(1):299. Epub 2016/09/28. doi: 10.1186/s13054-016-1478-z. PubMed PMID: 27670788; PubMed Central PMCID: PMCPMC5037640.

20. Soares RCM, Mattos LR, Raposo LM. Risk Factors for Hospitalization and Mortality due to COVID-19 in Espírito Santo State, Brazil. The American journal of tropical medicine and hygiene. 2020;103(3):1184-90. Epub 2020/07/20. doi: 10.4269/ajtmh.20-0483. PubMed PMID: 32682453; PubMed Central PMCID: PMCPMC7470570.

21. Alsofayan YM, Althunayyan SM, Khan AA, Hakawi AM, Assiri AM. Clinical characteristics of COVID-19 in Saudi Arabia: A national retrospective study. Journal of infection and public health. 2020;13(7):920-5. Epub 2020/06/15. doi: 10.1016/j.jiph.2020.05.026. PubMed PMID: 32534945; PubMed Central PMCID: PMCPMC7833063.

22. Byambasuren O, Cardona M, Bell K, Clark J, McLaws M-L, Glasziou PJOJotAoMM, et al. Estimating the extent of asymptomatic COVID-19 and its potential for community transmission: systematic review and meta-analysis. 2020;5(4):223-34.

23. Yang R, Gui X, Xiong Y. Comparison of Clinical Characteristics of Patients with Asymptomatic vs Symptomatic Coronavirus Disease 2019 in Wuhan, China. JAMA network open. 2020;3(5):e2010182. Epub 2020/05/28. doi:

10.1001/jamanetworkopen.2020.10182. PubMed PMID: 32459353; PubMed Central PMCID: PMCPMC7254178.

24. Cheng D, Calderwood C, Skyllberg E, Ainley A. Clinical characteristics and outcomes of adult patients admitted with COVID19 in East London: a retrospective cohort analysis. 2021;8(1):e000813. doi: 10.1136/bmjresp-2020-000813 \%J BMJ Open Respiratory Research.

25. Naser AY, Dahmash EZ, Alsairafi ZK, Alwafi H, Alyami H, Jalal Z, et al. Knowledge and Practices during the COVID-19 Outbreak in the Middle East: A Cross-Sectional Study. International journal of environmental research and public health. 2021;18(9). Epub 2021/05/01. doi: 10.3390/ijerph18094699. PubMed PMID: 33925024; PubMed Central PMCID: PMCPMC8125675.

26. Berenguer J, Ryan P, Rodríguez-Baño J, Jarrín I, Carratalà J, Pachón J, et al. Characteristics and predictors of death among 4035 consecutively hospitalized patients with COVID-19 in Spain. Clinical microbiology and infection : the official publication of the European Society of Clinical Microbiology and Infectious Diseases. 2020;26(11):1525-36. Epub 2020/08/08. doi: 10.1016/j.cmi.2020.07.024. PubMed PMID: 32758659; PubMed Central PMCID: PMCPMC7399713.

27. Nachtigall I, Lenga P, Jóźwiak K, Thürmann P, Meier-Hellmann A, Kuhlen R, et al. Clinical course and factors associated with outcomes among 1904 patients hospitalized with COVID-19 in Germany: an observational study. Clinical microbiology and infection : the official publication of the European Society of Clinical Microbiology and Infectious Diseases. 2020;26(12):1663-9. Epub 2020/08/22. doi: 10.1016/j.cmi.2020.08.011. PubMed PMID: 32822883; PubMed Central PMCID: PMCPMC7434317. 
28. Grasselli G, Greco M, Zanella A, Albano G, Antonelli M, Bellani G, et al. Risk Factors Associated With Mortality Among Patients With COVID-19 in Intensive Care Units in Lombardy, Italy. JAMA internal medicine. 2020;180(10):1345-55. Epub 2020/07/16. doi: 10.1001/jamainternmed.2020.3539. PubMed PMID: 32667669; PubMed Central PMCID: PMCPMC7364371 Getinge Group, Biotest, Draeger Medical Systems, Inc, Thermo Fisher Scientific, and Fisher \& Paykel outside the submitted work. Dr Zanella reported holding patents to WO2016189427 and WO2015IB55837 (licensed). Dr Bellani reported receiving grants and personal fees from Draeger Medical Systems, Inc, and Dimar SRL and personal fees from Hamilton Medical Products, Inc, Getinge Group, GE Healthcare, and Intersurgical outside the submitted work. Dr lotti reported receiving personal fees from Hamilton Medical Products, Inc, Intersurgical, Maquet Italia, Cinisello Balsamo Eurosets, and Burke \& Burke outside the submitted work. Dr Mojoli reported receiving fees for lectures from Hamilton Medical Products, Inc, GE Healthcare, and Seda SpA and a consultancy agreement between University of Pavia and Hamilton Medical Products, Inc. Dr Thompson reported receiving personal fees from Bayer AG outside the submitted work. Dr Pesenti reported receiving personal fees from Maquet Italia, Novalung/Xenios AG, Baxter International, Inc, and Boehringer Ingelheim outside the submitted work. Dr Cecconi reported receiving personal fees from Edwards Lifesciences, Directed Systems, and Cheetah Medical, Inc, outside the submitted work. No other disclosures were reported.

29. Zhang XB, Hu L, Ming Q, Wei XJ, Zhang ZY, Chen LD, et al. Risk factors for mortality of coronavirus disease-2019 (COVID19) patients in two centers of Hubei province, China: A retrospective analysis. PloS one. 2021;16(1):e0246030. Epub 2021/01/29. doi: 10.1371/journal.pone.0246030. PubMed PMID: 33507974; PubMed Central PMCID: PMCPMC7842894.

30. Shi C, Wang L, Ye J, Gu Z, Wang S, Xia J, et al. Predictors of mortality in patients with coronavirus disease 2019: a systematic review and meta-analysis. BMC infectious diseases. 2021;21(1):663. Epub 2021/07/10. doi: 10.1186/s12879-021-063690. PubMed PMID: 34238232; PubMed Central PMCID: PMCPMC8264491.

31. Henry BM, Aggarwal G, Wong J, Benoit S, Vikse J, Plebani M, et al. Lactate dehydrogenase levels predict coronavirus disease 2019 (COVID-19) severity and mortality: A pooled analysis. The American Journal of Emergency Medicine. 2020;38(9):1722-6. doi: https://doi.org/10.1016/j.ajem.2020.05.073.

32. Mejía F, Medina C, Cornejo E, Morello E, Vásquez S, Alave J, et al. Oxygen saturation as a predictor of mortality in hospitalized adult patients with COVID-19 in a public hospital in Lima, Peru. PloS one. 2020;15(12):e0244171. Epub 2020/12/29. doi: 10.1371/journal.pone.0244171. PubMed PMID: 33370364; PubMed Central PMCID: PMCPMC7769479.

33. Sulaiman T, Algharawi AA, Idrees M, Alzaidy RH, Faris K, Cullingford G, et al. The prevalence of gastrointestinal symptoms among patients with COVID-19 and the effect on the severity of the disease. JGH open : an open access journal of gastroenterology and hepatology. 2020;4(6):1162-6. Epub 2020/10/13. doi: 10.1002/jgh3.12415. PubMed PMID: 33043143; PubMed Central PMCID: PMCPMC7537315.

34. Tariq R, Saha S, Furqan F, Hassett L, Pardi D, Khanna S. Prevalence and Mortality of COVID-19 Patients With Gastrointestinal Symptoms: A Systematic Review and Meta-analysis. Mayo Clinic proceedings. 2020;95(8):1632-48. Epub 2020/08/06. doi: 10.1016/j.mayocp.2020.06.003. PubMed PMID: 32753138; PubMed Central PMCID: PMCPMC7284248. 
35. Bhatt AS, Jering KS, Vaduganathan M, Claggett BL, Cunningham JW, Rosenthal N, et al. Clinical Outcomes in Patients With Heart Failure Hospitalized With COVID-19. JACC Heart failure. 2021;9(1):65-73. Epub 2021/01/02. doi: 10.1016/j.jchf.2020.11.003. PubMed PMID: 33384064; PubMed Central PMCID: PMCPMC7833294.

36. Hussain Alsayed HA, Saheb Sharif-Askari F, Saheb Sharif-Askari N, Hussain AAS, Hamid Q, Halwani R. Early administration of remdesivir to COVID-19 patients associates with higher recovery rate and lower need for ICU admission: A retrospective cohort study. PloS one. 2021;16(10):e0258643. Epub 2021/10/27. doi: 10.1371/journal.pone.0258643. PubMed PMID: 34699552; PubMed Central PMCID: PMCPMC8547637.

37. Lai CC, Chen $\mathrm{CH}$, Wang $\mathrm{CY}$, Chen $\mathrm{KH}$, Wang YH, Hsueh PR. Clinical efficacy and safety of remdesivir in patients with COVID-19: a systematic review and network meta-analysis of randomized controlled trials. The Journal of antimicrobial chemotherapy. 2021;76(8):1962-8. Epub 2021/03/25. doi: 10.1093/jac/dkab093. PubMed PMID: 33758946; PubMed Central PMCID: PMCPMC8083728.

38. Dessie ZG, Zewotir T. Mortality-related risk factors of COVID-19: a systematic review and meta-analysis of 42 studies and 423,117 patients. BMC infectious diseases. 2021;21(1):855. doi: 10.1186/s12879-021-06536-3.

39. Parra-Bracamonte GM, Lopez-Villalobos N, Parra-Bracamonte FE. Clinical characteristics and risk factors for mortality of patients with COVID-19 in a large data set from Mexico. Annals of epidemiology. 2020;52:93-8.e2. Epub 2020/08/18. doi: 10.1016/j.annepidem.2020.08.005. PubMed PMID: 32798701; PubMed Central PMCID: PMCPMC7426229.

40. Rivera-Izquierdo M, Del Carmen Valero-Ubierna M, JL Rd, Fernández-García M, Martínez-Diz S, Tahery-Mahmoud A, et al. Sociodemographic, clinical and laboratory factors on admission associated with COVID-19 mortality in hospitalized patients: A retrospective observational study. PloS one. 2020;15(6):e0235107. Epub 2020/06/26. doi: 10.1371/journal.pone.0235107. PubMed PMID: 32584868; PubMed Central PMCID: PMCPMC7316360.

41. Camelo S, Latil M, Agus S, Dioh W, Veillet S, Lafont R, et al. A comparison between virus- versus patients-centred therapeutic attempts to reduce COVID-19 mortality. Emerging Microbes \& Infections. 2021;10(1):2256-63. doi: 10.1080/22221751.2021.2006579.

42. Challen R, Brooks-Pollock E, Read JM, Dyson L, Tsaneva-Atanasova K, Danon L. Risk of mortality in patients infected with SARS-CoV-2 variant of concern 202012/1: matched cohort study. 2021;372:n579. doi: 10.1136/bmj.n579 \%J BMJ.

43. Grint DJ, Wing K, Williamson E, McDonald HI, Bhaskaran K, Evans D, et al. Case fatality risk of the SARS-CoV-2 variant of concern B.1.1.7 in England, 16 November to 5 February. Euro surveillance : bulletin Europeen sur les maladies transmissibles = European communicable disease bulletin. 2021;26(11). Epub 2021/03/20. doi: 10.2807/1560-7917.Es.2021.26.11.2100256. PubMed PMID: 33739254; PubMed Central PMCID: PMCPMC7976383. 